\title{
Transcriptome analysis at four developmental stages of grape berry (Vitis vinifera cv. Shiraz) provides insights into regulated and coordinated gene expression
}

\author{
Crystal Sweetman ${ }^{1}$, Darren CJ Wong ${ }^{1}$, Christopher M Ford ${ }^{1}$ and Damian P Drew ${ }^{1,2^{*}}$
}

\begin{abstract}
Background: Vitis vinifera berry development is characterised by an initial phase where the fruit is small, hard and acidic, followed by a lag phase known as veraison. In the final phase, berries become larger, softer and sweeter and accumulate an array of organoleptic compounds. Since the physiological and biochemical makeup of grape berries at harvest has a profound impact on the characteristics of wine, there is great interest in characterising the molecular and biophysical changes that occur from flowering through veraison and ripening, including the coordination and temporal regulation of metabolic gene pathways. Advances in deep-sequencing technologies, combined with the availability of increasingly accurate $V$. vinifera genomic and transcriptomic data, have enabled us to carry out RNA-transcript expression analysis on a global scale at key points during berry development.
\end{abstract}

Results: A total of 162 million 100-base pair reads were generated from pooled Vitis vinifera (cv. Shiraz) berries sampled at 3-weeks post-anthesis, 10- and 11-weeks post-anthesis (corresponding to early and late veraison) and at 17-weeks post-anthesis (harvest). Mapping reads from each developmental stage (36-45 million) onto the NCBI RefSeq transcriptome of $23,720 \mathrm{~V}$. vinifera mRNAs revealed that at least $75 \%$ of these transcripts were detected in each sample. RNA-Seq analysis uncovered 4,185 transcripts that were significantly upregulated at a single developmental stage, including 161 transcription factors. Clustering transcripts according to distinct patterns of transcription revealed coordination in metabolic pathways such as organic acid, stilbene and terpenoid metabolism. From the phenylpropanoid/stilbene biosynthetic pathway at least 46 transcripts were upregulated in ripe berries when compared to veraison and immature berries, and 12 terpene synthases were predominantly detected only in a single sample. Quantitative real-time PCR was used to validate the expression pattern of 12 differentially expressed genes from primary and secondary metabolic pathways.

Conclusions: In this study we report the global transcriptional profile of Shiraz grapes at key stages of development. We have undertaken a comprehensive analysis of gene families contributing to commercially important berry characteristics and present examples of co-regulation and differential gene expression. The data reported here will provide an invaluable resource for the on-going molecular investigation of wine grapes.

Keywords: Grapevine, Illumina, Shiraz, RNA-seq, Transcriptome

\footnotetext{
* Correspondence: damian.drew@adelaide.edu.au

${ }^{1}$ Wine Science and Business, School of Agriculture Food and Wine, University

of Adelaide, Waite Campus, Urrbrae, South Australia 5064, Australia

${ }^{2}$ Department of Plant and Environmental Sciences, Faculty of Science,

University of Copenhagen, Frederiksberg 1871, Denmark
} 


\section{Background}

Berry development is a complex process displaying a double sigmoidal growth curve with three distinct phases, including two periods of growth separated by a lag phase during which expansion slows and seeds mature [1]. Cells are established in the first two weeks following flowering, and during the initial growth phase a rapid increase in berry size occurs as a result of cell expansion. The biosynthesis of tannins and hydroxycinnamates and several phenolic compound precursors takes place in the first growth phase [2], and organic acids accumulate in the vacuoles, with malic acid reaching a peak concentration before veraison and then decreasing throughout the second half of the growing season [3]. The short period known as veraison marks the boundary between the lag phase and the third phase of development, and is characterised by the initiation of sugar accumulation, a loss of photosynthetic capacity [4], and the rapid pigmentation of berries by anthocyanins in red grape varieties [1]. High levels of glucose and fructose accumulate after veraison while organic acid levels decrease; the resulting acid to sugar ratio present at harvest is one of the most important contributors to wine sensory characteristics [5]. Towards the end of this third phase of berry development, a number of compounds including terpenes, norisoprenoids, esters and thiols are synthesised [6]. The properties of the berry at harvest, including the final mix of primary and secondary metabolites that accumulate during ripening, are an important determinant of the quality, and therefore value, of the wine produced.

Although the biochemical and physical changes that occur during berry development are well characterised $[7,8]$, the biological processes that control them are less well understood. To a large extent, the biophysical changes that occur during the complex process of grape berry development must be influenced by the presence and activity of metabolic gene pathways. In turn, these metabolic pathways must be controlled by the transcriptional regulation of RNA. Understanding these pathways will give us a greater understanding of the fundamental processes that control berry development, and provide insights into the genetic basis of grape quality that could potentially benefit the wine industry. To this end, several studies have sought to investigate the transcriptional changes that occur during berry development using DNA microarrays [8-14]. Microarray analysis has also been used to investigate differences in gene expression between specific grape tissues [15], and in grapes exposed to a variety of biotic and abiotic stresses or imposed changes to growth conditions [16-23]. Additionally, a collection of microarrays has recently been combined with RNA sequencing to form a grapevine gene expression atlas [24]. The major limitation of most previous microarray studies is that they have generally been limited to interrogating only a portion of the total transcriptome. Many genes are not represented on the microarrays commonly used for grape analysis, while genes that exist in large and highly similar families may give ambiguous expression results due to non-specific hybridisation. Furthermore, the ability of probes to measure transcript abundance is constrained by the accuracy of sequences upon which the array was designed, which is particularly important given the high level of allelic variation in the $V$. vinifera species [25], and the genomic differences between commercial varieties.

The more recent microarray studies have benefited from substantial progress in defining the $V$. vinifera genome in the last five years. The genomes of the variety Pinot Noir and a Pinot Noir-derived variety named PN40024 have been sequenced by two consortia, providing an invaluable resource for studying the molecular mechanisms influencing grape development $[25,26]$. The $V$. vinifera genome, however, is highly complex and there have been difficulties in producing accurate genomic scaffolds due to its highly heterozygous nature [27]. The PN40024 variety was specifically bred to near-homozygosity to facilitate genomic sequencing and assembly, but most cultivated varieties are extremely heterozygous with allelic differences of up to $13 \%$ [25]. The difficulties in genomic assembly have been compounded by the relatively high number of transposons [28], and the fact that some gene families are highly repeated and interspersed with numerous pseudo genes [29]. Nevertheless, algorithmic predictions of the grapevine transcriptome, combined with a large amount of expressed sequence tag (EST) data, have been used to design and annotate microarray platforms for the interrogation of grape berry transcripts. Although valuable data on transcriptional regulation in grapes has been reported, the aforementioned technical limitations of microarrays have limited their level of coverage.

Massively parallel RNA deep-sequencing represents an alternative technological platform for investigating transcriptional regulation. It enables the precise elucidation of transcripts present within a particular sample, and can be used to calculate gene expression based on absolute transcript abundance [30]. In the single reported grapevine study to date, Zenoni et al. (2010) generated RNA sequencing data from Vitis vinifera (cv. Corvina), and provided an initial overview of the complex process of gene regulation during berry development [31]. Due to the rapidly advancing technology of next generation sequencing, the amount of sequencing data that can be generated in a single experiment has increased dramatically in recent years, as has the length of the sequencing reads. This has led to a greater level of transcriptome coverage and an increase in the specificity, and therefore accuracy, when mapping sequencing reads. Importantly, continuous incremental advances in defining the grapevine transcriptome in the form of functional annotation 
$[32,33]$ and gene ontology assignment [34] now enable an accurate description of the functional roles of the majority of $V$. vinifera genes. In this report, we use the latest RNA sequencing technology to carry out a comprehensive analysis of the global transcriptional profile of grape berries (cv. Shiraz) during the immature green phase, at early and late veraison, and in ripe berries. We investigate the suitability of a number of reference transcriptomes for RNA-Seq analysis in grapevine, validate a number of the transcriptional changes observed using quantitative real-time PCR, and describe the biological processes that are enriched in differentially regulated gene clusters.

\section{Results and discussion}

\section{Grape sampling and development}

Berries from $V$. vinifera (cv. Shiraz) were sampled at 7 to 14-day intervals throughout the growing season, with the shorter intervals occurring in the period coinciding with the expected time of veraison. Fruit development was monitored by the measurement of fresh weight and malic acid and tartaric acid content per berry in samples from 3 weeks post-anthesis until harvest at 17 weeks, and total soluble solids (degrees Brix; ${ }^{\circ} \mathrm{Bx}$ ) measurements were taken from 7 weeks until harvest. The fresh weight of berries increased throughout the season, with a slowing of growth observed at about 9 weeks followed by a rapid increase in fresh weight from 10 to 13 weeks. Malic acid content in berries increased early in the season and peaked at approximately 9 weeks post-anthesis (Figure 1A). From 9 to 12 weeks the malic acid content per berry dropped rapidly and continued to decrease until harvest. Total soluble solids, as measured by ${ }^{\circ} \mathrm{Bx}$, increased consistently between each sampling point, with the most rapid increase occurring between weeks 10 and 11 (Figure 1A). The end of the herbaceous plateau, decreasing malic acid content and rapidly increasing ${ }^{\circ} \mathrm{Bx}$ are examples of the physiological changes that characterise veraison, which is most easily recognised in red grape varieties by the development of pigment over a relatively short period of time (Figure 1B). Given our interest in the transcriptional changes that may be

A

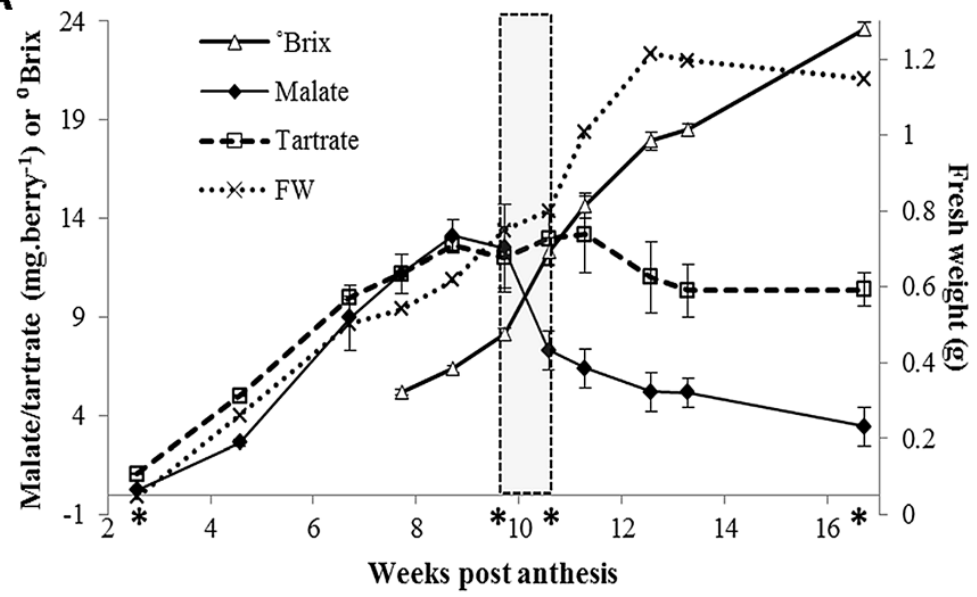

B

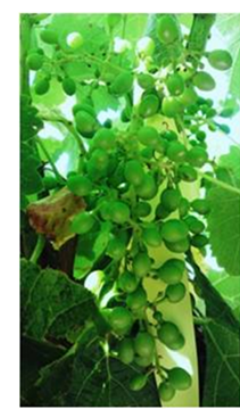

E-L 31

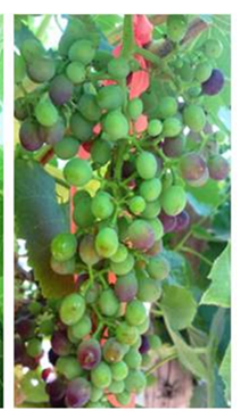

E-L 35

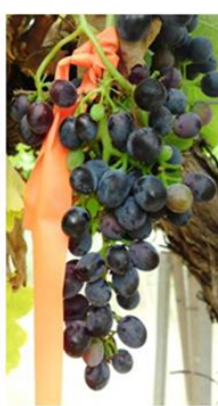

E-L 36

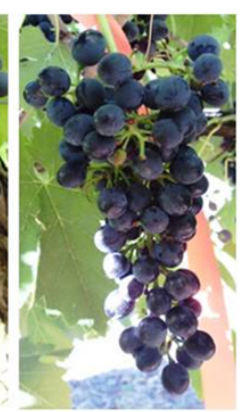

E-L 38

Figure 1 Shiraz berry developmental measurements. A. ${ }^{\circ}$ Brix, tartrate and malate levels are presented as the mean of three biological replicates $( \pm$ S.E.M.). Veraison is highlighted by a dashed box, and samples from which RNA was submitted for transcriptome sequencing are indicated with an asterisk below the $\mathrm{x}$-axis. B. Images of representative bunches at the time-points selected for sequencing, corresponding to developmental stages E-L 31, 35, 36 and 38 and referred to in the text as young berries, early-veraison, late-veraison and ripe berries. 
involved in regulating grape development, based on these data we chose to carry out global mRNA sequencing on samples from 3-, 10-, 11- and 17-weeks post-anthesis, corresponding to stage E-L 31, 35, 36 and 38 on the modified E-L system [35].

\section{Illumina HiSeq mRNA sequencing}

Prior to sequencing, RNA integrity numbers were determined for poly(A) mRNA isolated from each of the four developmental stages using a Bioanalyzer 2100 (Agilent Technologies). Calculated values of 9.20, 9.30, 8.60 and 9.30, respectively, indicated that little degradation of mRNA had occurred during extraction or subsequent processing, suggesting full-length or near full-length mRNAs were likely to be present and predominant. Each of the four mRNA samples was indexed with unique nucleic acid identifiers and sequenced on a single lane of an Illumina HiSeq 2000 instrument. In total, 162,353,167 reads of 100 bp were generated, giving a total of over 16 billion nucleotides of sequence data. This compares favourably with the 2.2 billion nucleotides of sequence data consisting of 59 million 36-44 bp reads obtained for the previous reported grape berry transcriptome sequencing project [31]. In addition to the almost 8-fold higher sequence coverage, the 3-fold longer read length enabled a much greater degree of accuracy when mapping to reference genomes or transcriptomes. De-multiplexing using the unique identifiers revealed that our data consisted of $35,656,501$ reads from young berries, $39,624,765$ reads from pre-veraison berries, 42,052,446 reads from post-veraison berries and 45,019,455 reads from ripe berries. This provided almost 10-fold higher sequencing read number than a recent Illumina-based transcriptome analysis of fruit development in Chinese bayberry, which investigated gene regulation based on 5.3 million 90 bp reads [36].

\section{Investigation of mapping references for RNA-Seq analysis}

We first investigated a number of reference transcript collections in order to determine whether a comprehensive and accurate description of berry transcriptional profiles could be developed by mapping and counting the reads generated through Illumina sequencing against predicted mRNA transcripts. Two independent groups have generated near-complete $V$. vinifera (cv. Pinot Noir and cv. PN40024) consensus genome assemblies [25,26], and the former of these groups, the French-Italian Public Consortium for Grapevine Genome Characterization, has released two publically accessible versions of the complete $V$. vinifera genome at $8 \mathrm{x}$ and $12 \mathrm{x}$ coverage (available from http://www.genoscope.cns.fr/externe/Download/ Projets/Projet_ML/data/) [25]. Algorithmic predictions of mRNA transcriptomes based on this data and using the GAZE computational framework resulted in the prediction of 30,434 or 26,346 transcripts from the $8 x$ and $12 x$ genome assemblies, respectively, and provided the first two datasets to which we mapped and counted our sequencing reads. In addition, the National Center for Biotechnology Information reference sequence (NCBI RefSeq) database provided an alternative resource of predicted $V$. vinifera mRNA transcripts [33]. While NCBI RefSeq transcripts are based on the 12x genome of Jaillon et al. (2007), they are predicted by the Gnomon algorithm, which draws on supporting evidence such as ESTs and alignments to orthologous transcripts and proteins, and are manually curated and continually updated [37]. The NCBI RefSeq nucleotide collection, consisting of 23,720 annotated transcripts, comprised the third reference dataset for our mapping reference.

The use of an mRNA transcript collection as a mapping reference is an alternative approach to that taken by Zenoni et al. (2010), who instead used the draft consensus genome reported by Jaillon et al. (2007) as a reference. Mapping of mRNA sequencing reads against genomic scaffolds requires prior knowledge of gene structure, or can be carried out through the use of algorithmic predictions of splice junctions [38]. However, given the complexity of the draft consensus genome, its high reported heterozygosity, and the difference in grape variety under investigation, we chose instead to focus on the transcribed component for our analysis. When carrying out RNA-Seq mapping, we excluded reads with greater than two ambiguous nucleotides, as well as the small proportion of reads that were less than $60 \mathrm{bp}$ in length. This resulted in a total pool of 148,945,405 reads from the four developmental stages that were counted for transcript mapping (Table 1). We used a similarity threshold of $98 \%$, and set the minimum proportion of the read that must match a reference at 0.5 to allow for the mapping of reads that included up to $50 \mathrm{bp}$ of UTR in cases where this was not included as part of the mapping reference. Somewhat surprisingly only about $58.0 \%$ of our sequencing reads could be mapped against the Genoscope $8 \mathrm{x}$ or $12 \mathrm{x}$ predicted transcriptomes (Table 1). In contrast, $83.6 \%$ could be mapped to the NCBI RefSeq collection. The proportion of our sequencing reads mapping to the NCBI RefSeq collection is actually higher than the proportion of shorter reads that were previously mapped to the Vitis vinifera draft consensus genomic scaffolds [31], highlighting the suitability of our chosen mapping reference.

Although the use of a nucleotide mapping reference means the genes investigated in our analysis are determined by pre-existing transcriptomic data, the high percentage of reads mapped under high-stringency conditions indicated a high level of coverage of actual transcribed sequences. Additionally, the use of the NCBI RefSeq nucleotide collection facilitates direct comparison of mapped transcripts with well-described gene functions and 
Table 1 Comparison of transcriptome datasets as a reference for RNA-Seq analysis

\begin{tabular}{lllll}
\hline Developmental stage & Counted reads & $\begin{array}{l}\text { Genoscope 8x } \\
\text { (30434 transcripts) }\end{array}$ & $\begin{array}{l}\text { Genoscope 12x } \\
\text { (26346 transcripts) }\end{array}$ & $\begin{array}{l}\text { NCBI RefSeq } \\
\text { (23720 transcripts) }\end{array}$ \\
\hline Young berries & 32283153 & $17127563(53.0)$ & $16340511(50.6)$ & $24972894(77.3)$ \\
Early-veraison & 36280465 & $21111334(58.2)$ & $22423774(56.6)$ & $30695559(84.6)$ \\
Late-veraison & 38434765 & $23572898(61.3)$ & $22522747(58.6)$ & $33605499(87.4)$ \\
Ripe berries & 41947022 & $24606233(59.6)$ & $25516490(58.1)$ & $35251865(85.4)$ \\
Total & 148945405 & $86418028(58.0)$ & $86803522(58.3)$ & $124525817(83.6)$ \\
\hline
\end{tabular}

Number of Illumina HiSeq sequencing reads from each developmental stage mapped to selected reference mRNA transcript collections. The percentage of counted reads that were mapped is presented in parenthesis.

manually updated annotations. The method employed by Bellin and co-workers [23], whereby pyrosequencing of 3' cDNA ends and de novo contig assembly was used to create a library of unigenes for microarray design, represents an approach to transcriptome analysis that overcomes the issue of predetermined transcript data. This combination of next generation sequencing and microarray generation will be particularly valuable for non-model species for which genomic information is limited. However, in the case of $V$. vinifera, for which relatively well-annotated genomic and transcriptomic data are available, the use of a nucleotide mapping reference represents a convenient technique that allows the utilisation of annotations detailed and updated on NCBI.

While the Genoscope 8x predicted transcriptome contained 30,434 sequences, the NCBI RefSeq dataset consisted of only 23,720 sequences. Given that a much higher proportion of our Illumina sequencing reads mapped to the RefSeq dataset than to the Genoscope dataset ( $83.6 \%$ compared to $58 \%$; Table 1), it was considered unlikely that the difference of almost 7,000 transcripts was simply a result of absent genes from the RefSeq mRNA collection. A batch BLAST search using each of the Genoscope predicted transcripts as a query against the RefSeq mRNA dataset revealed that about 28,000 (92\%) of the 30,434 Genoscope transcripts had a hit in the RefSeq dataset with an e-value approaching zero (data not shown). However, this included numerous duplicates where multiple short Genoscope transcripts were matched to a single RefSeq transcript. When these duplicates were removed, a list of approximately 20,000 accessions remained. Furthermore, investigation of a subset of Genoscope transcripts that had no BLAST hit within the RefSeq transcript collection revealed that many of these predicted transcripts were 100 nucleotides or less, and were probably partial gene sequences that did not have a significant match because of their length. We therefore propose that the widely used $V$. vinifera transcriptome prediction from Genoscope contains multiple redundant accessions that have probably come about as a result of incorrect assignment of splice junctions. This analysis, combined with a higher proportion of mapped sequence reads, indicated that the manually curated NCBI RefSeq dataset is the most comprehensive and accurate collection of $V$. vinifera mRNAs currently available, and we proceeded to use this set of reference transcripts to investigate mRNA abundance and transcriptional regulation in grape berries.

\section{Transcript expression analysis}

Transcript abundance was determined by the calculation of Reads Per Kilobase of exon per Million mapped reads (RPKM) [30]. Unique reads were counted to matching transcripts, and non-specifically mapped reads were allocated on a proportional basis relative to the number of unique reads already mapped. A limitation of this method is in the case of differentiating between recently duplicated isogenes with coding sequence exceeding $98 \%$ identity, and thus expression values in these instances should not be considered definitive. A method of measuring differences in expression between highly similar isogenes by microarray analysis of non-coding regions has been described for a subset of the $V$. vinifera genome [9]. The accuracy of the RPKM method for calculating transcript expression is also impacted in cases where full-length sequences are not transcribed due to premature stop codons, structural variation or differences between the mapping reference and the actual transcript. Nevertheless, with these limitations in mind, out of the 23,720 predicted transcripts in the NCBI RefSeq mRNA collection, 17,942-18,729 transcripts could be detected at each developmental stage (Table 2). For these data the lower limit for detection was designated to be an RPKM of 0.5 , or if the RPKM value was less than 0.5 then a minimum of five uniquely matched reads (at greater than 98\% identity over $100 \mathrm{bp}$ ) were required for a transcript to be considered present. Only 3,208 out of 23,720 transcripts, approximately $13.5 \%$, did not meet these criteria for detection in any of the four developmental stages (Additional file 1: Table S2). To put the RPKM values from our study in perspective, a value of 0.5 corresponds to an average transcript coverage of 2, or about $2000 \mathrm{bp}$ of sequencing read coverage for a $1000 \mathrm{bp}$ transcript. In a recent comparable study, Zenoni et al. (2010) estimated that their statistical analysis would be reliable when applied to genes with 6 
Table 2 Transcript abundance measurements at each developmental stage

\begin{tabular}{lcccc}
\hline & Young berries & Early-veraison & Late-veraison & Ripe \\
\hline RPKM > 200 & 679 & 477 & 466 & 411 \\
RPKM 10-200 & 7458 & 8179 & 7582 & 7908 \\
RPKM 0.5-10 & 8994 & 7994 & 8016 & 8327 \\
RPKM 0-0.5 (unique reads > 5) & 1499 & 1717 & 1878 & 2083 \\
Total detected & 18720 & 18367 & 17942 & 18729 \\
\hline
\end{tabular}

Numbers of transcripts from the NCBI Vitis vinifera RefSeq dataset detected at various levels of abundance at each time-point, as calculated by reads per kilobase of exon per million reads (RPKM).

mapped 36-44 bp reads covering 200 bp of transcript, which could otherwise be expressed as average coverage of approximately one [31]. A benefit of mapping $100 \mathrm{bp}$ reads compared with the shorter reads generated in the work of Zenoni et al. (2010) is the increased specificity, and thus accuracy, of transcript expression analysis. Allowing for two mismatches, a 36 bp read maps with only $94 \%$ identity, inevitably leading to alignment with multiple locations, especially in the case of closely related multi-gene families. In the current study, approximately $85 \%$ of reads that were matched at $98 \%$ identity or greater were aligned to a single location in our reference dataset (data not shown), compared with $66.6 \%$ matched to unique locations by Zenoni et al. (2010) [31].

The fact that $70-80 \%$ of the NCBI RefSeq mRNA transcripts for $V$. vinifera could be detected in each of our samples, and $86.5 \%$ of transcripts could be detected in at least one sample, is a testament to the power of RNASeq analysis as a technique for transcriptional studies, compared with microarray analyses in which probes have historically covered a limited portion of the $V$. vinifera transcriptome. Furthermore, the depth of our Illumina sequencing data enabled us to investigate the expression of transcripts that are present at extremely variable absolute levels. For example, the lower limit for detection for which we report transcript regulation in this study, corresponding to an RPKM of 0.5 (Table 2), represents transcripts with an absolute abundance 90,000fold lower than the most abundant transcript in ripe berries, XM_002284998.2, which had an RPKM of 44,999. The mRNA XM_002284998.2 (corresponding to Genoscope accession GSVIVT00020222001), which encodes an uncharacterised proline-rich protein of 236 amino acids, with sequence similarity to extensin related cell-wall proteins, accounted for an impressive $4.5 \%$ of the sequencing reads generated from ripe berries. This highlights one of the benefits of RNA-Seq expression analysis over microarray analysis in uncovering transcripts that may be of interest. Microarrays determine changes in the relative expression of transcripts between two or more samples, but do not provide accurate quantitative data on the absolute level of expression of a transcript within any given RNA sample due to differences in probe binding specificity and efficiency. As a resource for grapevine researchers, we present the absolute expression levels of all transcripts in each of the four developmental stages investigated here in Additional file 1: Table S1, alongside the closest matching Genoscope accession and the functional annotation of the encoded protein.

\section{Global comparison with microarray analysis of developing grape}

Given the surfeit of literature reporting transcript expression in grapes based on the microarray platform, we investigated the correlation between our measurements of mRNA transcript abundance based on RNA-Seq analysis and gene expression levels previously reported at equivalent developmental stages based on the Affymetrix GeneChip. Deluc et al. (2007) investigated transcriptional regulation in developing grapes of Vitis vinifera (cv. Cabernet Sauvignon and cv. Chardonnay) at a number of developmental stages, including those corresponding to E-L 31, E-L 35, E-L 36 and E-L 38 [8]. Although the varieties of grapes investigated by Deluc et al. (2007) differed from the variety studied in this report, we predicted that a majority of transcripts should exhibit similar relative abundances within each stage of berry development investigated here. For this comparison, we considered only GeneChip probesets for which the originating EST has an exact BLASTn match (evalue $=0$ ) in the NCBI RefSeq dataset, and discarded probesets that cross-hybridised with multiple transcripts. Transcripts that were expressed at low or background level in either microarray or RNA-Seq analysis were also removed, leaving 6899 and 6848 transcripts for Cabernet Sauvignon and Chardonnay, respectively. For this subset of transcripts, the correlation between our RNA-Seq analysis and their expression in the corresponding developmental stages reported by Deluc et al. (2007) was approximately $\rho=0.73$ for Cabernet Sauvignon and $\rho=$ 0.72 for Chardonnay (Figure 2A). These relatively high correlation coefficients indicate that the absolute transcript expression levels we report within a single developmental stage of berry based on RNA-Seq give similar results to previous data generated by the Affymetrix GeneChip microarray. 
We also examined the correlation between the pattern of relative expression formed by the four developmental stages examined in our RNA-Seq analysis compared with the equivalent expression pattern as measured by microarray [8]. We found that the expression patterns of a majority of transcripts in both Cabernet Sauvignon and Chardonnay were positively correlated with our Shiraz RNA-Seq analysis. This included about 30\% of transcripts for which the patterns of expression measured by the two platforms were extremely highly correlated with a $\rho \geq 0.9$ (Figure 2B). The majority of transcript expression patterns were positively correlated to some degree, with about $57 \%$ of transcripts exhibiting a medium to high correlation of $\rho \geq 0.6$. The high correlation between differential expression of transcripts reported here and the expression patterns previously measured by microarray, goes some way towards validating the utility of our data for investigating transcriptional regulation during grape development.

\section{Highly expressed transcripts throughout grape development}

Approximately 400-700 transcripts from each developmental stage had an RPKM value of 200 or greater (Table 2), and as such represented the top 1.7-2.9\% of mRNAs by absolute expression level. Of these transcripts, 153 had an RPKM of over 200 in all four samples under investigation (Additional file 1: Table S2). In addition to 31 uncharacterised proteins, the products of these highly expressed transcripts included a number of proteins that would generally be expected to be highly expressed in most cell types. These included 16 ribosomal proteins, 12 translation initiation and elongation factors, 8 proteins involved in amino acid metabolism, 6 glycolysis pathway enzymes, 2 catalase isoforms, 2 actinrelated proteins, 2 vacuolar proton ATPases, super-oxide dismutase and RuBisCo. It is interesting to note that 147 out of 153 of these highly expressed transcripts have a matching Affymetrix probeset ID, despite the fact that only $34 \%$ of the RefSeq sequences are represented on the microarray. This is likely due to the fact that the design of microarray probesets was based predominantly on EST data, in which highly expressed transcripts are inherently over-represented. Conversely, of the 3,208 transcripts that were not detected in any of our four samples, only 239 (7.5\%) are represented by an Affymetrix probe (Additional file 1: Table S3).

Given the apparent constitutively high level of transcription for the genes mentioned above, it could be suggested that they are likely to play an important role in biological processes occurring during berry development. However, since we have not investigated other tissue from grapevine in this study, we do not present evidence that the transcripts are specifically involved in

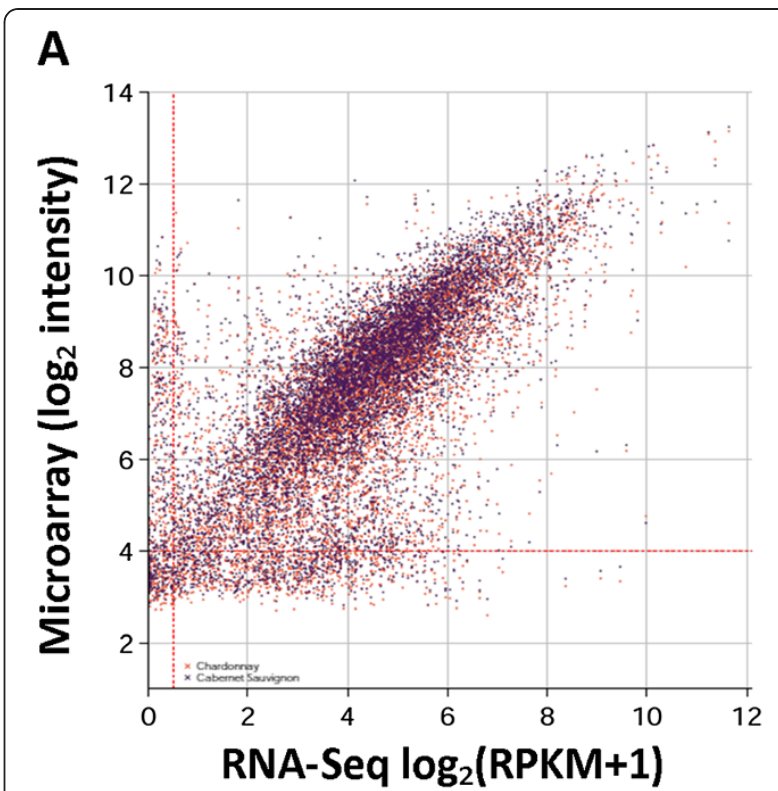

B

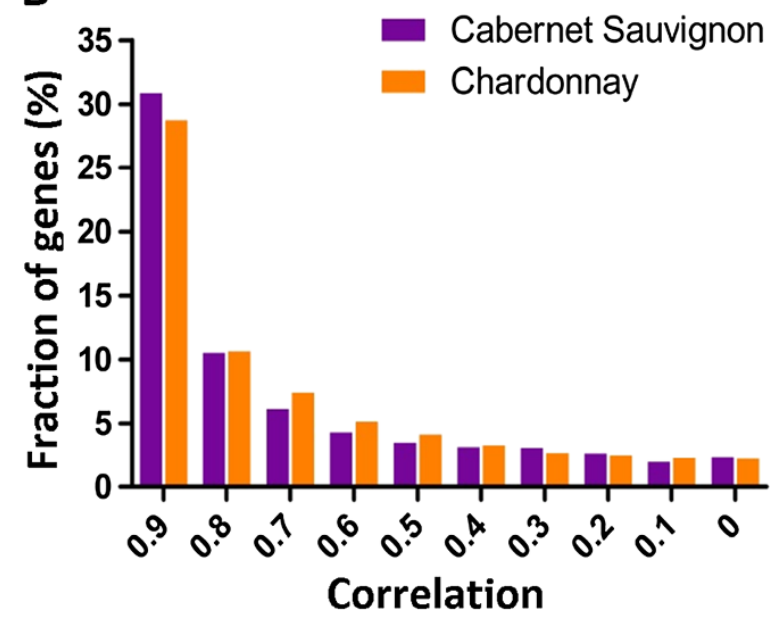

Figure 2 Global comparison of RNA-Seq and microarray analysis of transcript expression in developing grape. A. Comparison of microarray probeset intensities for developing Cabernet Sauvignon (purple) and Chardonnay (orange) [8] with transcript abundance for the corresponding genes measured in our study as expressed by log2 (RPKM + 1). Expression values charted here consists of the mean of four developmental stages corresponding to E-L 31, E-L 35, E-L 36 and E-L 38, and give Spearman correlation coefficients of $\rho=0.73$ and $\rho=0.72$ for Cabernet Sauvignon and Chardonnay, respectively. Dashed lines represent the cut-off whereby genes are not considered expressed in either platform and are not included in the calculation of correlation coefficients. B. Histogram showing the distribution of correlated genes during berry development. Mapped transcripts having a Spearman correlation between correlation thresholds were counted from a total of 7189 unique transcripts measured by both platforms. 
berry development relative to other grapevine tissues. Nevertheless, some potential berry-related genes can be seen within this list. Since high levels of malic acid are synthesized in berries until veraison, and both the concentration and absolute level rapidly decrease after veraison [3], it is not surprising that a cytoplasmic malate dehydrogenase (MDH; XM_002278672.2), which catalyses the reversible conversion of oxaloacetate to malate, was highly abundant at all stages. Another functionally annotated cytoplasmic MDH enzyme (XM_0022786002.2) was similarly abundant, while the third isoform (XM_002277507.2) was approximately 50-fold less abundant. Immediately adjacent to $\mathrm{MDH}$ in the citric acid cycle, citrate synthase (XM_002278145.2) was also one of the consistently abundant transcripts in our data. Given that malate accumulates in the berry vacuole where it is compartmentally separated from the citric acid cycle enzymes, the highly abundant putative malate carrier protein (XM_002285686.1) may warrant further functional investigation.

Another abundant, putatively berry-specific gene was a chalcone synthase isoform (CHS2; XM_002263983.1), which is a potential upstream regulator of a number of phenolic secondary metabolites, including tannins, anthocyanins and flavonols. In contrast, CHS1 has previously been shown to be developmentally regulated with highest expression occurring in young berries [39], a result that is consistent with our RNA-Seq analysis (Additional file 1: Table S1). The finding that two genes putatively involved in the metabolism of alpha-linolenic acid (XM_002272955.2 and XM_002285538.2) were highly abundant is interesting since $n-3$ fatty acids such as linolenic have only been found to be present in grapes at extremely low concentrations [40]. Thus, the high expression level of these two transcripts in grapes suggests further characterisation may be required to determine their true functional activity. One isoform of hydroxymethylgutarylCoA synthase (XM_002282398.2) was highly abundant, while the other (XM_002262655.2) was not detected in any of our samples, highlighting the importance of isoformspecific expression data.

\section{Specifically up-regulated transcripts}

We used our quantitative expression analysis to investigate genes that are transcriptionally regulated during specific stages of berry development. First, we investigated genes that were more highly expressed in a single developmental stage when compared with their expression levels in each of the other three stages. To account for low and zero values in our data while still identifying biologically significant changes, differences were calculated relative to an RPKM of 0.1 when calculating fold changes from RPKM values of less than 0.1. Thus, the difference between 0.02 and 1.00 was considered a 10fold or greater increase, but not a 50-fold increase. This went some way towards discarding unrealistically high expression changes that are an unavoidable consequence of data that incorporates values approaching and including zero. In total, there were 4,185 transcripts that exhibited 3-fold or greater increased expression at a single developmental stage compared with all other stages (Table 3). A relatively low number of these transcripts were specifically up-regulated at early- or late-veraison (194 and 59 transcripts, respectively). The low number of genes specifically regulated at these time points is probably due to the fact that only a single week separated the two samples. Furthermore, in order to capture a representative biological selection of transcripts at each time-point, RNA for Illumina sequencing was purified from tissue consisting of 20 berries collected from 10 bunches that had been monitored from the beginning of the growing season and tagged at 50\% cap-fall (see Methods). Since it takes approximately one week for a single bunch to develop from $0 \%$ to $100 \%$ cap-fall, it could be argued that individual grapes on any given bunch are separated by up to a week in their absolute developmental age. This biological variation within each of our early- and late- veraison stages could have masked transcriptional regulation events that take place over the relatively short one-week period during which pigmentation occurs (Figure 2, E-L 35 to E-L 36). Also, it has been reported that major changes in gene expression can occur over as little as 24 hours, and that this happens before changes in $\mathrm{pH}$, sugars and berry colouring can be observed [9]. Therefore, an in-depth analysis of genes that are differentially expressed between young berries and veraison, and between veraison and fullripening, could yield more useful information about global changes in metabolism. With this in mind, we also generated data on the number of transcripts that are specifically up-regulated at both the time-points taken around veraison (E-L 35 and E-L 36), compared with their expression level in young or ripe berries (Table 3 'veraison'). A complete list of all 4,185 transcripts that are specifically up-regulated 3 -fold or more at a single developmental stage corresponding to the transcripts counted in Table 3, and an additional 122 transcripts that are specifically over-expressed during both earlyand late-veraison, is provided in Additional file 2.

Given the observed similarity between RPKM data from early- and late-veraison samples, we investigated the overall correlation between these two stages in order to estimate the technical variation within our experiment. The Pearson's correlation coefficient for global transcript expression between these two stages (E-L 35 and E-L 36) was $\rho=0.99$, which is equivalent to the correlation expected for high quality technical replicates of the same RNA sample [41]. Additionally, only about 2\% of genes exhibited a $\log _{2}$ transcript abundance difference 
Table 3 Transcripts over-expressed at a single developmental stage

\begin{tabular}{|c|c|c|c|c|c|c|}
\hline & Young berries (E-L 31) & Early-veraison (E-L 35) & Late-veraison (E-L 36) & Harvest (E-L 38) & Total & Veraison \\
\hline$>50$-fold higher & 282 & 1 & 0 & 65 & 348 & 3 \\
\hline 10-50 fold higher & 789 & 31 & 1 & 175 & 996 & 60 \\
\hline 3-10 fold higher & 1814 & 162 & 58 & 807 & 2841 & 312 \\
\hline Total & 2885 & 194 & 59 & 1047 & 4185 & 375 \\
\hline
\end{tabular}

The numbers of transcripts significantly up-regulated in berries at a single developmental stage relative to all other samples. Fold changes are calculated compared with a minimum RPKM value of 0.1. Given the similarity in transcript expression patterns between early and late-veraison (E-L 35 and E- $L$ 36), the relative expression of transcripts in both of these stages compared with E-L 31 and E-L 38 are reported in the separated column "veraison".

of greater than 1.6 (equating to about 3 -fold) between these stages (data not shown), which could be explained as a conservative description of the transcripts truly differentially expressed between early- and late-veraison. Thus, although it will be desirable to analyse global transcript abundance from more highly separated timepoints around veraison when investigating developmental regulation in future studies, we were able to use these two samples as de facto replicates in order to demonstrate that our RPKM expression data was reproducible and that 3-fold and greater changes in abundance were very unlikely to be the result of technical variation.

One of the clearest findings from an analysis of transcripts that were highly over-expressed at a single stage was the large number of biological processes activated in young berries (stage E-L 31) that do not occur during veraison or in ripe berries. In young berries, 2,885 of the 23,720 investigated transcripts were specifically overexpressed relative to all other time-points, while 1,047 were specifically upregulated in ripe berries (Table 3). The 2,885 transcripts that were at least 3-fold up-regulated in immature berries represented a significant $12 \%$ of the total grapevine predicted transcriptome, or approximately $15 \%$ of the transcripts expressed in berries. A portion of these genes were extremely highly up-regulated with 282 transcripts upregulated over 50 -fold in young berries compared with expression levels in any other sample. Many of the transcripts more highly expressed in young berries can be linked with the photosynthetic capacity of grapes during early stages of development, which decreases dramatically during ripening [4]. For example, 18 of the 20 annotated chlorophyll a-b binding proteins from grapevine were amongst these 2,885 transcripts, as were 12 out of 15 photosystem I reaction center subunit-encoding transcripts, two of the three transcripts encoding the photosystem II reaction center W and transcripts for photosystem II $5 \mathrm{kDa}$ and $22 \mathrm{kDa}$ corecomplex proteins (data is searchable in Additional file 2). Transcripts encoding enzymes from other metabolic pathways reported to occur early in grape berry development were also highly over represented in the list, such as genes involved in the biosynthesis of tannin precursors. These include anthocyanidin reductase, leucanthocyanidin reductase, and five anthocyanidin 3-O-glucosyltransferases, which stabilise anthocyanins through glycosylation [42].
Transcription factors are of particular interest given their ability to control the expression of numerous genes, and thus their ability to regulate biological pathways and developmental processes. There were 26 annotated transcription factors specifically over-expressed 50 -fold or greater in young berries, most of which had zero or negligible expression at the other stages investigated here (Table 4). These included nine transcripts encoding ethylene-responsive transcription factor (ERF) 5-like proteins. We found that a further four ERF5-like transcripts were specifically up-regulated between 10and 50-fold in young berries (Additional file 2). Combined, these transcripts comprised 13 of the 17 annotated ERF5-like genes, while the remaining four ERF5-like transcripts were all up-regulated 2- to 3-fold in young berries. Six other transcription factors that were 50 -fold or greater up-regulated in young berries are annotated as ethyleneresponsive transcription factors, including two ERF17s, ERF7, ERF23, ERF109 and ERF-WIN1 (Table 4). Whether these families of transcription factors are responsive to ethylene in grapes has not been established, and it is important to remember that the majority of functional annotations are made based on sequence similarity to proteins from other species, predominantly Arabidopsis. Indeed, while ethylene signalling is known to play an important role in the ripening of climacteric fruit, the precise role of ethylene signalling, if any, in grape development remains an active area of research $[43,44]$. Nevertheless, the high degree of transcriptional specificity of these families of transcription factors is a strong indication that they are responsible for regulating biological processes that occur early during grape berry development.

While all of the transcription factors that were 50 -fold or greater specifically up-regulated in a single sample were found in young berries, four transcription factors were specifically over-expressed at least 3 -fold during veraison, and 29 were specifically over expressed at least 3 -fold in ripe berries (Table 4). One veraison-specific transcription factor is of particular interest due to its similarity to, and thus functional annotation as, an UPBEAT1 gene. The UPBEAT1 transcription factor has been shown to control the transition from cell proliferation to cell differentiation in Arabidopsis roots by modifying the balance of reactive oxygen species [45]. In grapes, an oxidative burst has been 
Table 4 Specifically up-regulated transcription factors

\begin{tabular}{|c|c|c|c|c|c|c|c|}
\hline RefSeq Accession & $\begin{array}{l}\text { Closest } \\
\text { Genoscope match }\end{array}$ & $\begin{array}{l}\text { Affymetrix } \\
\text { Probeset ID }\end{array}$ & $\begin{array}{l}\text { Young } \\
\text { berries }\end{array}$ & $\begin{array}{l}\text { Early- } \\
\text { veraison }\end{array}$ & $\begin{array}{l}\text { Late- } \\
\text { veraison }\end{array}$ & $\begin{array}{l}\text { Ripe } \\
\text { berries }\end{array}$ & $\begin{array}{l}\text { Encoded protein } \\
\text { annotation }\end{array}$ \\
\hline XM_002282012.2 & GSVIVT00014253001 & & 125.18 & 0.51 & 0.76 & 0.35 & $\begin{array}{l}\text { Ethylene-responsive Transcription } \\
\text { factor } 5\end{array}$ \\
\hline XM_002281930.1 & GSVIVT00014247001 & & 121.59 & 1.73 & 1.03 & 0.29 & $\begin{array}{l}\text { Ethylene-responsive Transcription } \\
\text { factor } 5\end{array}$ \\
\hline XM_002282133.2 & GSVIVT00036589001 & & 114.34 & 0.52 & 0.60 & 0.45 & $\begin{array}{l}\text { Ethylene-responsive Transcription } \\
\text { factor ERF109 }\end{array}$ \\
\hline XM_002276536.2 & GSVIVT00016398001 & 1608812_at & 105.10 & 0.15 & 0.27 & 0.13 & $\begin{array}{l}\text { Ethylene-responsive Transcription } \\
\text { factor ERF017-like }\end{array}$ \\
\hline XM_002282279.1 & GSVIVT00023866001 & & 90.51 & 0.24 & 0.22 & 0.86 & $\begin{array}{l}\text { Ethylene-responsive Transcription } \\
\text { factor 7-like }\end{array}$ \\
\hline XM_002281911.2 & GSVIVT00014244001 & & 83.05 & 0.51 & 0.47 & 0.14 & $\begin{array}{l}\text { Ethylene-responsive Transcription } \\
\text { factor } 5 \text {-like }\end{array}$ \\
\hline XM_002281777.2 & GSVIVT00014237001 & 1613698_at & 80.37 & 1.36 & 0.50 & 0.28 & $\begin{array}{l}\text { Ethylene-responsive Transcription } \\
\text { factor } 5\end{array}$ \\
\hline XM_002281895.2 & GSVIVT00014242001 & 1619600_at & 73.43 & 0.80 & 0.53 & 0.19 & $\begin{array}{l}\text { Ethylene-responsive Transcription } \\
\text { factor } 5\end{array}$ \\
\hline XM_002268377.2 & GSVIVT00000349001 & & 46.99 & 0.24 & 0.15 & 0.06 & $\begin{array}{l}\text { Ethylene-responsive Transcription } \\
\text { factor WIN1-like }\end{array}$ \\
\hline XM_002281047.2 & GSVIVT00022870001 & 1616185_at & 39.00 & 0.11 & 0.03 & 0.17 & Transcription factor bHLH96-like \\
\hline XM_002282131.1 & GSVIVT00014256001 & & 38.48 & 0.44 & 0.23 & 0.13 & $\begin{array}{l}\text { Ethylene-responsive Transcription } \\
\text { factor } 5\end{array}$ \\
\hline XM_002280334.1 & GSVIVT00032308001 & & 36.98 & 0.00 & 0.00 & 0.05 & $\begin{array}{l}\text { Ethylene-responsive Transcription } \\
\text { factor ERF017 }\end{array}$ \\
\hline XM_002281876.2 & GSVIVT00014240001 & & 31.92 & 0.20 & 0.40 & 0.10 & $\begin{array}{l}\text { Ethylene-responsive Transcription } \\
\text { factor } 5\end{array}$ \\
\hline XM_002281835.2 & GSVIVT00014238001 & 1613799_at & 29.46 & 0.30 & 0.09 & 0.00 & $\begin{array}{l}\text { Ethylene-responsive Transcription } \\
\text { factor } 5\end{array}$ \\
\hline XM_002284201.1 & GSVIVT00014754001 & & 29.28 & 0.35 & 0.21 & 0.20 & Transcriptional activator Myb \\
\hline XM_002263558.1 & GSVIVT00006679001 & & 22.72 & 0.12 & 0.00 & 0.03 & Transcription factor RAX1 \\
\hline XM_002263958.2 & GSVIVT00008628001 & & 19.81 & 0.04 & 0.00 & 0.04 & $\begin{array}{l}\text { Ethylene-responsive Transcription } \\
\text { factor ERF023 }\end{array}$ \\
\hline XM_002268533.2 & GSVIVT00000129001 & & 18.68 & 0.08 & 0.07 & 0.16 & Transcription factor TCP15-like \\
\hline XM_002283709.1 & GSVIVT00032414001 & 1609286_at & 18.29 & 0.13 & 0.05 & 0.11 & GATA Transcription factor 9 \\
\hline XM_002274170.1 & GSVIVT00034800001 & & 11.10 & 0.04 & 0.00 & 0.03 & Transcriptional activator Myb \\
\hline XM_002276513.1 & GSVIVT00037009001 & & 9.52 & 0.08 & 0.03 & 0.13 & Transcription factor bHLH135 \\
\hline XM_003633976.1 & GSVIVT00014248001 & & 8.02 & 0.00 & 0.09 & 0.03 & $\begin{array}{l}\text { Ethylene-responsive Transcription } \\
\text { factor 5-like }\end{array}$ \\
\hline XM_002283058.1 & GSVIVT00020927001 & 1613614_at & 7.56 & 0.11 & 0.00 & 0.00 & Transcription factor bHLH135 \\
\hline XM_002276926.1 & GSVIVT00029219001 & & 7.43 & 0.00 & 0.00 & 0.00 & Transcription repressor MYB4 \\
\hline XM_002274226.2 & GSVIVT00018597001 & & 5.91 & 0.07 & 0.00 & 0.06 & Transcription factor bHLH118-like \\
\hline XM_002284800.1 & GSVIVT00014836001 & & 5.31 & 0.07 & 0.04 & 0.04 & Heat stress Transcription factor B-4 \\
\hline XM_003632349.1 & GSVIVT00001240001 & 1621346_at; & 0.10 & 50.36 & 25.69 & 7.32 & $\begin{array}{l}\text { B3 domain-containing transcription } \\
\text { factor ABI3-like }\end{array}$ \\
\hline XM_002275111.1 & GSVIVT00025350001 & & 0.14 & 30.00 & 15.82 & 4.91 & Transcription factor HBP-1 b(c1)-like \\
\hline XM_003632364.1 & & & 0.00 & 4.66 & 4.85 & 0.89 & Transcription factor UPBEAT1-like \\
\hline XM_002283723.2 & & & 0.00 & 2.52 & 0.93 & 0.06 & $\begin{array}{l}\text { myb family transcription factor } \\
\text { APL-like }\end{array}$ \\
\hline XM_002272753.2 & GSVIVT00031144001 & 1609798_at & 30.57 & 50.29 & 34.79 & 155.93 & Trihelix transcription factor GTL2-like \\
\hline XM_002276158.2 & GSVIVT00017225001 & 1610832_at & 8.91 & 19.82 & 17.88 & 67.73 & $\begin{array}{l}\text { Probable WRKY transcription } \\
\text { factor } 32\end{array}$ \\
\hline
\end{tabular}


Table 4 Specifically up-regulated transcription factors (Continued)

\begin{tabular}{|c|c|c|c|c|c|c|c|}
\hline$\overline{\text { XM_002281158.1 }}$ & GSVIVT00028232001 & & 5.78 & 13.73 & 18.95 & 61.77 & $\begin{array}{l}\text { Probable WRKY transcription factor } \\
\text { 47-like }\end{array}$ \\
\hline XM_003635597.1 & & 1611921_at & 7.36 & 16.93 & 13.95 & 59.56 & GATA transcription factor 26-like \\
\hline XM_002269660.2 & GSVIVT00025898001 & & 1.14 & 9.70 & 11.70 & 57.63 & WRKY transcription factor 6-like \\
\hline XM_002273307.2 & GSVIVT00013494001 & 1620116_at & 9.62 & 13.68 & 10.78 & 44.93 & GATA transcription factor 26 \\
\hline XM_002275540.1 & GSVIVT00002773001 & 1607465_at & 1.34 & 2.26 & 3.46 & 43.23 & $\begin{array}{l}\text { Probable WRKY transcription } \\
\text { factor } 57\end{array}$ \\
\hline XM_002274248.2 & GSVIVT00033300001 & & 0.22 & 0.67 & 1.28 & 23.91 & $\begin{array}{l}\text { Ethylene-responsive transcription } \\
\text { factor ERF003 }\end{array}$ \\
\hline XM_003631122.1 & GSVIVT00030611001 & 1608728_at & 6.36 & 6.37 & 4.36 & 19.42 & $\begin{array}{l}\text { Heat stress transcription factor } \\
\text { A-8-like }\end{array}$ \\
\hline XM_002267778.1 & GSVIVT00006201001 & 1609629_at & 0.76 & 0.23 & 0.36 & 10.56 & $\begin{array}{l}\text { Ethylene-responsive transcription } \\
\text { factor ERF113 }\end{array}$ \\
\hline XM_002283591.1 & GSVIVT00024804001 & & 0.10 & 0.88 & 1.21 & 9.53 & $\begin{array}{l}\text { Ethylene-responsive transcription } \\
\text { factor RAP2-11 }\end{array}$ \\
\hline XM_002275357.2 & GSVIVT00003416001 & 1622116_at & 0.59 & 0.46 & 0.10 & 4.36 & $\begin{array}{l}\text { Transcription factor bHLH144 } \\
\text { isoform } 2\end{array}$ \\
\hline XM_003632808.1 & GSVIVT00034227001 & & 0.14 & 0.88 & 0.45 & 4.20 & Transcription factor bHLH87-like \\
\hline XM_002280888.1 & & 1618136_at & 0.38 & 0.14 & 0.16 & 4.13 & $\begin{array}{l}\text { Ethylene-responsive transcription } \\
\text { factor ERF114-like }\end{array}$ \\
\hline XM_002274180.2 & GSVIVT00033298001 & 1609559_at & 0.00 & 0.05 & 0.19 & 3.98 & $\begin{array}{l}\text { Ethylene-responsive transcription } \\
\text { factor ERF003 isoform } 1\end{array}$ \\
\hline XM_002272053.1 & GSVIVT00003403001 & & 1.02 & 0.19 & 0.23 & 3.18 & $\begin{array}{l}\text { Probable WRKY transcription } \\
\text { factor } 28\end{array}$ \\
\hline XM_002279376.2 & GSVIVT00030359001 & 1618408_at & 0.38 & 0.09 & 0.06 & 2.89 & Transcription factor bHLH75 \\
\hline XM_002275834.2 & GSVIVT00037958001 & & 0.59 & 0.34 & 0.19 & 2.80 & $\begin{array}{l}\text { Ethylene-responsive transcription } \\
\text { factor ERF113-like }\end{array}$ \\
\hline XM_002285559.1 & GSVIVT00015050001 & & 0.15 & 0.53 & 0.06 & 2.36 & Transcription factor bHLH93 \\
\hline XM_002279450.1 & GSVIVT00016545001 & & 0.03 & 0.04 & 0.02 & 2.32 & $\begin{array}{l}\text { Putative transcription factor } \\
\text { bHLH041 }\end{array}$ \\
\hline XM_002284180.2 & GSVIVT00025614001 & & 0.07 & 0.08 & 0.02 & 1.99 & $\begin{array}{l}\text { Heat stress transcription factor } \\
\text { B-3-like }\end{array}$ \\
\hline XM_002264354.2 & GSVIVT00007519001 & & 0.17 & 0.00 & 0.00 & 1.36 & $\begin{array}{l}\text { Ethylene-responsive transcription } \\
\text { factor ERF098-like }\end{array}$ \\
\hline XM_002270623.2 & GSVIVT00029005001 & & 0.02 & 0.04 & 0.02 & 1.34 & $\begin{array}{l}\text { Probable WRKY transcription } \\
\text { factor } 72\end{array}$ \\
\hline XM_002267757.2 & GSVIVT00006494001 & 1607431_at & 0.00 & 0.12 & 0.00 & 1.04 & $\begin{array}{l}\text { Probable WRKY transcription factor } \\
\text { 53-like }\end{array}$ \\
\hline XM_002279303.1 & GSVIVT00020055001 & & 0.00 & 0.00 & 0.00 & 0.96 & Heat stress transcription factor A-6b \\
\hline XM_003633801.1 & GSVIVT00020889001 & & 0.00 & 0.19 & 0.17 & 0.96 & $\begin{array}{l}\text { AP2-like ethylene-responsive } \\
\text { transcription factor AlL5-like }\end{array}$ \\
\hline XM_002279882.1 & GSVIVT00032269001 & & 0.00 & 0.05 & 0.00 & 0.71 & Transcription factor WER \\
\hline XM_002277185.2 & GSVIVT00020895001 & & 0.05 & 0.02 & 0.02 & 0.68 & $\begin{array}{l}\text { Probable WRKY transcription } \\
\text { factor } 72\end{array}$ \\
\hline XM_002274351.1 & GSVIVT00037881001 & & 0.00 & 0.00 & 0.00 & 0.62 & $\begin{array}{l}\text { Probable WRKY transcription } \\
\text { factor } 45\end{array}$ \\
\hline
\end{tabular}


observed during veraison and is accompanied by the modulation of numerous of ROS scavenging enzymes, including peroxidases, peroxiredoxins, thioredoxins and glutathione-S-transferases [11]. Since many of the transcripts for these enzymes were shown to increase at veraison, the $V$. vinifera UPBEAT1-like transcription factor, XM_003632349.1, could be a potential target for further investigation. In ripe berries, the WRKY family of transcription factors was the most over represented, with 9 out of 58 putative members in grapevine specifically expressed in this sample. WRKY-type transcription factors have previously been implicated in pathogen response pathways in grapes $[46,47]$, and given that berries are most likely to suffer from fungal attack during late ripening stages, the highly regulated expression patterns reported here could be a further indication that some WRKY-type transcription factors are activated in response to biotic stress. Six members of the ethylene-responsive transcription factor family were also up-regulated in ripe berries, although only the ERF113 sub-family was represented by more than a single transcript (Table 4 ).

\section{An overview of gene ontology enrichment during berry development}

In addition to describing transcripts that were highly upregulated at a single developmental stage, transcripts that exhibited differential expression between a number of time points were investigated using statistical clustering. This technique revealed transcripts from the pool of differentially regulated genes that exhibited similar patterns of expression over the four developmental stages investigated here, regardless of the absolute level of expression. We present 10 clusters of developmentally regulated genes comprising 8,948 transcripts that displayed some degree of differential expression (Figure 3). In agreement with our finding that a large number of transcripts were specifically over expressed in young berries, two of the largest clusters contained transcripts upregulated in the first developmental stage. Cluster 1 contained 2,545 transcripts that were highly specific to young berries, while cluster 9 contained 1,227 transcripts that were most highly abundant in young berries and exhibited decreasing abundance in later stages. Cluster 10 (413 transcripts) also contained genes that were most abundant in young berries and decreased through to harvest, and cluster 5 (905 transcripts) contained genes that were up-regulated in young and ripe berries, but were less abundant around veraison. The majority of the transcripts reported as specifically upregulated in young berries based on 3-fold or greater RPKM changes (Table 3 and Additional file 2) fell within clusters 1 and 9. Also consistent with our analysis of stage specific up-regulation presented in Table 3, only 349 and 203 transcripts were specifically up-regulated at either early- or late-veraison, respectively (clusters 2 and $3)$, while 653 transcripts were up-regulated at both veraison stages (cluster 6). Cluster 4 consisted of 1,133 transcripts that were strongly up-regulated in ripe berries, while cluster 7 (629 transcripts) and cluster 8 (889 transcripts) contained genes for which expression increased throughout development and peaked in ripe berries.

In order to produce a global description of biological processes enriched in each cluster of similarly regulated transcripts, we generated an overview of gene ontology (GO) terms using AgriGO [34]. The AgriGO GO analysis tool retrieved descriptions of gene function based on the standardised vocabulary of the Gene Ontology bioinformatics initiative [48]. We then used the recentlycreated REVIGO web server to summarise these long lists of GO terms by removing redundant terms and grouping related terms based on semantic similarity [49]. Because GO terms have been assigned using BLAST, Pfam and Interpro scans, individual annotations should be viewed with caution. Nevertheless, for large groups of genes, statistically enriched terms can give insights into biological pathways that are likely to be highly active by comparing them to the frequency at which those GO terms appear in the whole transcriptome. A number of enriched ontological terms were reported several times amongst our clustered transcripts that relate to biological processes which could be expected to be enriched in developing fruit. For example, transcripts annotated with the GO terms "cellular reproductive process" and "post-embryonic development" were found to be enriched in six and five separate gene clusters, respectively (Figure 3). Given that statistical enrichment is calculated in comparison to the whole transcriptome, it is not surprising that GO terms relating to embryo development and reproduction were consistently enriched in berries in general. A more specifically enriched subset of GO terms were those relating to photosynthesis. These were enriched in cluster 1 only, which included transcripts that were highly upregulated in young green berries compered to berries at veraison and harvest, and is in agreement with our initial observation that many transcripts involved in photosynthesis were specifically expressed at this stage. Similarly, GO terms relating to thylakoid membrane localisation were enriched in cluster 10 , which consisted of genes that had decreasing abundances throughout development. These results confirm that the gene ontology enrichment detailed here describes biologically relevant metabolic events occurring at different stages of berry development.

An analysis of cluster 4 indicates that secondary metabolic pathways in general were highly up-regulated in ripe berries, as was the biosynthesis of modified amino acids, aromatic compounds, and phenylpropanoids (Figure 3 ). The most statistically significant enrichment within our cluster analysis was of transcripts 


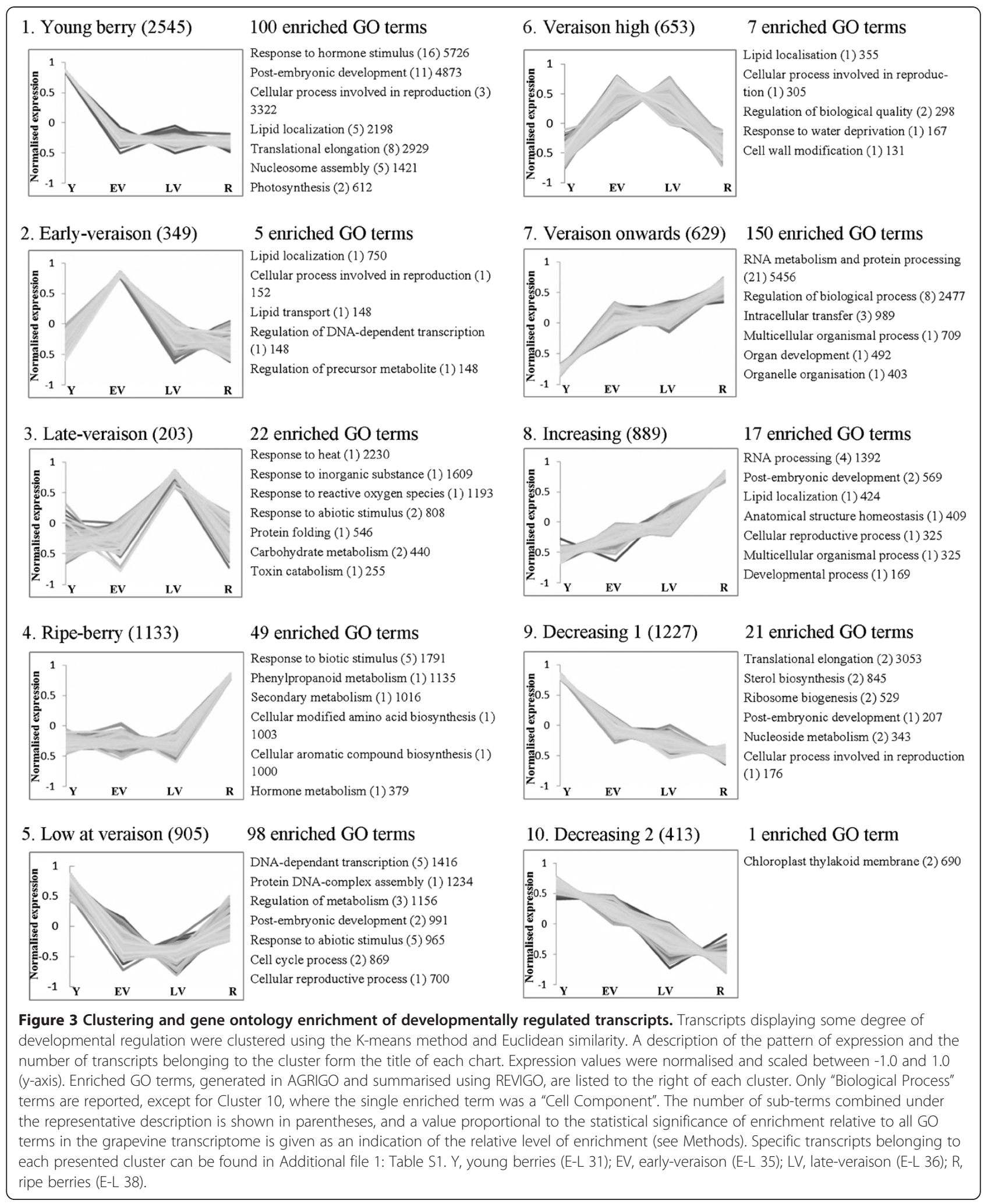


involved in responding to hormone signalling, which were highly enriched in cluster 1 . This could suggest that overall, hormone-controlled metabolic pathways are most likely to be activated in the early stages of grape development. Additionally, since terms relating to ribosome biogenesis, nucleosome assembly and translational elongation are enriched in clusters 1 and 9, it appears that berries were more translationally active during early development than they were later in the season. This could be explained by the high rate of cell division and differentiation occurring in the weeks following flowering, which later decreases as berry growth increasingly comes about through cell expansion and vacuolar enlargement $[1,50]$. The GO term "response to heat" was significantly enriched in cluster 3 (late veraison). An indepth analysis of transcripts located in this cluster revealed that 27 heat shock proteins were present, comprising approximately $13 \%$ of the genes in cluster 3 , and representing more than one third of all annotated heat shock proteins in $V$. vinifera (Additional file 1). We subsequently found that the minimum temperature on the morning of grape collection at late-veraison was $20.8^{\circ} \mathrm{C}$, compared with $12.8-13.7^{\circ} \mathrm{C}$ on other days of collection and also that the maximum temperature on the day prior to sample collection was the highest of the growing season at $37.8^{\circ} \mathrm{C}$ (data not shown). Given the wellcharacterised role of a number of heat shock proteins in response to environmental stimuli such as heat, water stress and oxidative stress [51], this is most likely an example of highly coordinated transcript regulation in response to environmental stimulus, rather than an example of developmental regulation.

A method that has been used previously for the ontological description of grapevine genes is GO-slim, which utilises a simplified subset of GO terms to give a broad overview of ontological content, but assigns many transcripts into vague categories such as "cellular process" or "other biological process" [31]. The descriptive summaries of GO term enrichment generated here using the AgriGO and REVIGO web tools represent a significant advance over previous techniques for ontological description of gene clusters. However, since our analysis of differential transcript expression has been carried out on samples from a specific vineyard over a single growing season, it cannot be inferred that the patterns of transcript expression, and therefore of metabolic pathway activation, are definitively linked with developmental changes. While it is likely that developmentally regulated transcripts have been identified, it is also possible that specific environmental, biotic or abiotic conditions that existed at the time of sampling have played a part in differential transcript regulation. Nevertheless, the differential regulation of transcripts in selected metabolic pathways that was observed during this season will be discussed below, and our full RPKM-based transcript abundance and cluster analyses are detailed in Additional file 1.

\section{Organic acid metabolism}

The berry metabolism of organic acids including malate, tartrate and ascorbate is an area of active research because of their contribution to juice and wine acidity and to the organoleptic characteristics and ageing potential of wine [3]. Additionally, the malate concentration of harvested berries can affect malolactic acid fermentation and influence the growth of malolactic bacteria [52]. Despite the clear developmentally regulated pattern of malate accumulation and degradation (Figure 1a), the majority of genes encoding enzymes directly involved in malate metabolism, including malate dehydrogenase $(\mathrm{MDH})$ and $\mathrm{NAD}(\mathrm{P})$-dependant malic enzyme, were expressed at all four stages of development investigated, with little differential regulation. Two exceptions to this were isoforms of cytoplasmic MDH (XM_002278600.2) and mitochondrial malic enzyme (XM_002266661.2), which were allocated to cluster 9 and thus decreased through berry development, although transcript abundance remained relatively high (Table 5). Since these two enzymes are involved in malate biosynthesis from oxaloacetate or pyruvate, respectively, their decreasing expression could be reflected in the observed physiological decrease in malate. The constitutive expression of other $\mathrm{MDH}$ and malic enzyme isoforms is likely due to the involvement of malic acid in numerous facets of plant primary metabolism, including the tricarboxylic acid cycle and the glyoxylate pathway [3]. In contrast to malate biosynthesis genes, all three transcripts encoding phosphoenolpyruvate carboxykinases (PEPCK; XM_003635567.1, XM_003635619.1 and XM_003635634.1) were allocated to cluster 7 (most highly expressed from veraison onwards), and two transcripts encoding PEP carboxylases (PEPC; XM_002280533.2 and XM_002280806.1) were in cluster 10 (decreasing expression). PEPCK enzymes catalyse the conversion of oxaloacetate to PEP, while PEPC carries out the reverse reaction. Thus, since $\mathrm{MDH}$ enzymes catalyse the reversible interconversion of oxaloacetate and malate, the potential decrease in oxaloacetate in mature berries caused by altered expression of PEPC and PEPCK could influence malate degradation by shifting the function of MDH enzymes towards malate catabolism. One isoform each of PEPCK (XM_003635567.1) and PEPC (XM_002280533.2) were included in the qRT-PCR validation of our RNA-Seq analysis, and it was shown that the expression differences observed between the four developmental stages were consistent across three biological replicates (Figure 4). Since the catabolism of malate can only occur when the acid is accessible to metabolic enzymes outside the vacuole, the compartmentation of 
Table 5 Organic acid metabolism

\begin{tabular}{|c|c|c|}
\hline Encoded protein description & Cluster & RefSeq accession(s) \\
\hline \multirow[t]{2}{*}{ Malate dehydrogenase } & 9 (decreasing) & XM_002278600.2 \\
\hline & NC & $\begin{array}{l}\text { XM_002265044.2, XM_002284873.2, XM_002283583.1, XM_002278676.2, } \\
\text { XM_002277507.2, XM_002263634.2, XM_003631644.1, XM_002275406.2, } \\
\text { XM_002285320.2 }\end{array}$ \\
\hline \multirow[t]{3}{*}{ Malic enzyme } & 9 (decreasing) & XM_002266661.2 \\
\hline & NC & XM_002265729.2, XM_002283715.1, XM_002283778.2,XM_003631725.1 \\
\hline & ND & XM_003631423.1 \\
\hline \multirow[t]{2}{*}{ Phosphoeno/pyruvate carboxylase } & 10 (decreasing) & XM_002280533.2, XM_002280806.1 \\
\hline & NC & XM_002285405.1 \\
\hline \multirow[t]{2}{*}{ Phosphoenolpyruvate carboxykinase } & 7 (veraison onwards) & XM_003635567.1, XM_003635619.1, XM_003635634.1 \\
\hline & NC & XM_003632437.1 \\
\hline Tonoplast dicarboxylate transporter & 6 (veraison up-regulated) & XM_003635577.1, XM_002277749.1 \\
\hline \multirow[t]{3}{*}{ GDP-Mannose-3,5-epimerase } & 1 (young berry) & XM_002279341.2 \\
\hline & 9 (decreasing) & XM_002283862.2 \\
\hline & 10 (decreasing) & XM_003631951.1 \\
\hline \multirow[t]{2}{*}{ GDP-L-galactose phosphorylase (VTC2) } & 1 (young berry) & XM_002278303.2 \\
\hline & NC & XM_002263621.1 \\
\hline Galactose dehydrogenase & 1 (young berry) & XM_002270526.2 \\
\hline L-galactono-1,4-lactone dehydrogenase & NC & XM_002274178.2 \\
\hline \multirow[t]{2}{*}{ L-idonate dehydrogenase } & 1 (young berry) & XM_002267626.2, XM_002269900.2 \\
\hline & 7 (increasing) & XM_002269859.2 \\
\hline \multirow[t]{2}{*}{ Galacturonic acid reductase } & 5 (low at veraison) & XM_002285191.1 \\
\hline & 7 (veraison onwards) & XM_002285183.2 \\
\hline
\end{tabular}

malate may also influence rates of its accumulation and degradation during berry development. Tonoplast dicarboxylate transporters (TDTs) have been shown to be responsible for the active transport of malate into plant vacuoles [53], and their genomic disruption in Arabidopsis led to decreased malate accumulation [54]. The two transcripts encoding TDTs in grapevine (XM_002277749.1 and XM_003635577.1) were allocated to cluster 7 (highest expression at veraison) and decreased 20 -fold between veraison and harvest, and the expression pattern of the latter was confirmed by qRT-PCR (Figure 4). A decrease in malate transport into the vacuole between veraison and harvest, combined with the action of cytoplasmic MDH and PEPCK in malate catabolism, could explain the developmental pattern of malate accumulation and degradation observed in $V$. vinifera.

Ascorbate is the main soluble antioxidant in plants and is predominantly synthesised in green tissues by the well-characterised Smirnoff-Wheeler pathway, in which the direct ascorbate precursor L-galactono-1,4-lactone is produced from GDP-L-mannose by the sequential action of GDP-mannose-3,5-epimerase (GME), GDP-L-galactose phosphorylase (VTC2), L-galactose-1-phosphate phosphatase and L-galactose dehydrogenase (L-GalDH) [55,56]. A more recently proposed alternative pathway for ascorbate biosynthesis involves the production of L-galactono-1,4-lactone from $\mathrm{D}$-galacturonic acid by the enzyme galacturonic acid reductase (GalUR) [57]. In a final step, L-galactono1,4-lactone is converted to ascorbate by L-galactono-1,4lactone dehydrogenase (GLDH). As a central component of redox metabolism in plants, ascorbate exists in equilibrium with its oxidised form dehydroascorbate, which can be catabolised to oxalate and L-threonate as well as being recycled to ascorbate. The ascorbate catabolic pathway that is of most interest to grape researchers, however, is its conversion into tartrate via an L-idonate intermediate; a pathway in which only one enzyme, L-idonate dehydrogenase (L-IdnDH), has been biochemically characterised [58]. In our data, three isoforms of GME (XM_002279341.2, XM_002283862.2 and XM_003631951.1) were allocated to clusters 1,9 and 10, indicating that they were specifically expressed in young berries, or were most abundant in young berries and then decreased during ripening (Table 5). Similarly, the single isoform of L-GalDH (XM_002270526.2) and the most abundant isoform of VTC2 (XM_002278303.2) were allocated to cluster 1. Also, although GLDH (XM_002274178.2) was not differentially expressed enough to be allocated a cluster in our analysis, its abundance did decrease during development and was 3 -fold lower in ripe berries than in immature 


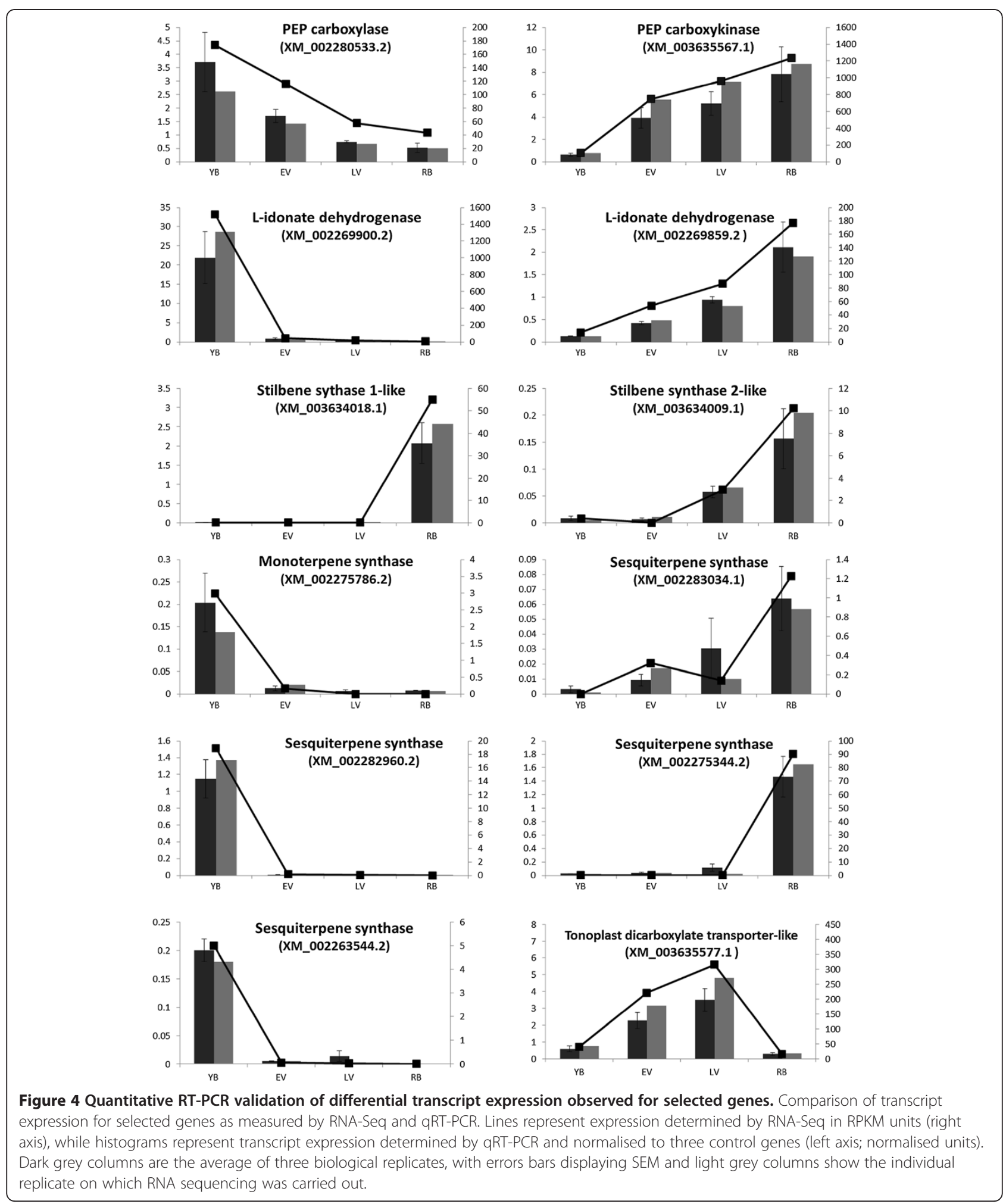


berries (Additional file 1). Given that both ascorbate and tartrate levels have been shown to increase in grape berries most rapidly from about two weeks after flowering until veraison [56], our data suggests that this is potentially controlled transcriptionally through differential expression of components of the Smirnoff-Wheeler pathway. Comparable results were obtained for genes of this pathway investigated with quantitative real-time polymerase chain reaction and reported by Melino et al. (2009). The transcript most similar to the characterised GaluR from strawberry (XM_002285191.1) was detected at very low levels in young berries, and not at all in the other samples. However, several other transcripts encoding putative oxidoreductases that are also homologues of GalUR were expressed at much higher levels, including XM_002285183.2, which was allocated to cluster 7 (veraison onwards). Two of the three potential L-IdnDH isoforms were specific to young berries and located in cluster 1 (XM_002267626.2 and XM_002269900.2) while a third isoform (XM_002269859.2) was in cluster 7, suggesting that the biosynthesis of tartrate from ascorbate may be controlled at different stages of grape development by different genes (Table 5). The transcript expression levels for two LIdnDH isofroms, XM_002269900.2 and XM_002269859.2, were validated by $\mathrm{qRT}-\mathrm{PCR}$, and demonstrated that the patterns were consistent across three replicates from different vines (Figure 4).

\section{Co-regulation of phenylpropanoid/stilbene biosynthetic genes}

A grape secondary metabolite that has received a great deal of attention in recent times is the polyphenolic compound resveratrol (3,5,4'-trihydroxy-trans-stilbene). Resveratrol is a phytoalexin involved in pathogen defence in grapevine [59], although it has also been shown to be present in healthy grapes [60]. Resveratrol is found in red wine and can positively regulate a number of beneficial physiological processes in animals [61]. The resveratrol biosynthesis pathway consists of four enzymes that sequentially transform phenylalanine into this specialised secondary metabolite. The first three enzymes, phenylalanine ammonia lyase (PAL), cinnamic acid 4hydroxylase $(\mathrm{C} 4 \mathrm{H})$ and 4-coumarate:CoA ligase (4CL), are components of the common phenylpropanoid pathway, which also leads to the production of phenolic compounds such as lignins, anthocyanins and other flavonoids. The fourth enzyme, stilbene synthase (STS), exists only in plants that produce stilbenes, and can catalyse the final step by converting 4-coumaroyl-CoA and three molecules of malonyl-CoA into cis- or trans-resveratrol. Although this biosynthetic pathway is commonly described as comprising four single enzymes, each are encoded by multigene families, which have potentially redundant activities, and may have different temporal or spatial expression.
The NCBI annotation of proteins encoded by the RefSeq mRNA transcripts suggests that there are 12 PALs, 3 C4Hs, 12 CLs and a startling 38 STSs. Despite the fact that less than $35 \%$ of the RefSeq mRNAs were differentially expressed enough to be included in our cluster analysis, almost all the transcripts in the stilbene biosynthesis pathway were assigned to a cluster. This confirms the well-reported observation that both general phenylpropanoid metabolism and specialised resveratrol metabolism are highly regulated processes in grapes. The majority of PAL, C4H and STS transcripts were grouped in cluster 4, indicating they were specifically up-regulated in ripe berries. In contrast, the $4 \mathrm{CL}$ transcripts exhibited more varied expression patterns, including three transcripts in cluster 1 (young fruit), two in cluster 5 (low at veraison) and one each in clusters 8 (increasing), 9 and 10 (decreasing; Table 6). A PAL transcript has previously been reported to be up-regulated early in the season under water-deficit, as measured by the intensity of the GeneChip probeset 1613113_at (corresponding to XM_0022272890.1) [62]. This particular transcript was the only one of 12 putative grapevine PAL genes which was not assigned a cluster in our analysis, and therefore the specific co-regulation of the majority of PAL genes in ripe berries that we saw in our data was not observed in that study. Guillaumie et al. (2011) reported that two PAL isoforms, corresponding to XM_002281763 and XM_002267917, increased in abundance over the final week of ripening, which is consistent with our results for these transcripts [13]. The first three steps of the phenylpropanoid pathway provide 4coumaroyl CoA as a substrate for chalcone synthase (CHS), which produces chalcone as the precursor for the important organoleptic flavonoids and anthocyanins. While seven potential CHS transcripts are annotated in the RefSeq mRNA collection, three of these were not detected in our data and may represent $V$. vinifera genes expressed in tissue other than grape. Two of the four detectable CHS transcripts were grouped in cluster 1 (XM_002276885.2 and XM_002276910.1), one was in cluster 5 (XM_002263983.1), and one was not differentially regulated (XM_002276617.1).

Several $V$. vinifera STSs have been shown biochemically to be involved in resveratrol biosynthesis [63-65], however the high sequence similarity amongst this multi-gene family (85-99\% identity) suggests they may all carry out a similar, or identical, biochemical reaction. Thus, an accurate description of the expression of each isoform is required for a full understanding of the conditions and tissue in which resveratrol is likely to be produced. Our data indicated that 36 of the 38 STSs were co-regulated in cluster 4 , one was not detected, and one was in cluster 8 , which also consisted of genes most highly expressed at harvest (Table 6). A high proportion of reads mapped to each RefSeq STS transcript 
Table 6 Phenylpropanoid/stilbene pathway transcripts

\begin{tabular}{|c|c|c|}
\hline Encoded protein description & Cluster & RefSeq accession(s) \\
\hline \multirow[t]{5}{*}{ Phenylalanine ammonia-lyase } & 1 (young berry) & XM_002285241.1 \\
\hline & 2 (early veraison) & XM_002278480.2 \\
\hline & 4 (ripe berry) & $\begin{array}{l}\text { XM_002268220.2, XM_002267917.2, XM_003633939.1, XM_002268145.2, } \\
\text { XM_003633937.1, XM_003633938.1, XM_002268737.2, XM_002268696.2 }\end{array}$ \\
\hline & 5 (low at veraison) & XM_002281763.2 \\
\hline & NC & XM_002272890.1 \\
\hline \multirow[t]{2}{*}{ Cinnamic acid 4-hydroxylase } & 4 (ripe berry) & XM_002266106.1, XM_002266001.1 \\
\hline & 5 (low at veraison) & XM_002266202.1 \\
\hline \multirow[t]{6}{*}{ 4-coumarate:CoA ligase } & 1 (young berry) & XM_002285884.2,XM_002285885.1, XM_002274958.2 \\
\hline & 5 (low at veraison) & XM_002265509.1, XM_002272746.2 \\
\hline & 8 (increasing) & XM_002270324.1 \\
\hline & 9 (decreasing) & XM_002279486.2, XM_002270556.1 \\
\hline & NC & XM_002276317.2 \\
\hline & ND & XM_002271550.2, XM_002269909.1, XM_002266436.2 \\
\hline Stilbene synthase 1-like & 4 (ripe berry) & $\begin{array}{l}\text { XM_002264419.2, XM_002263926.1a, XM_002263845.2, XM_003634014.1a, } \\
\text { XM_002263686.2, XM_003634018.1, XM_003634015.1, XM_003634017.1 }\end{array}$ \\
\hline \multirow[t]{2}{*}{ Stilbene synthase 2-like } & 4 (ripe berry) & $\begin{array}{l}\text { XM_002265955.1, XM_002278447.2, XM_002278349.1, XM_002265193.2, } \\
\text { XM_002271335.2, XM_002268806.2b, XM_003634020.1b, XM_002272093.2, } \\
\text { XM_003634032.1 }\end{array}$ \\
\hline & 8 (increasing) & XM_003634009.1 \\
\hline Stilbene synthase 4-like & 4 (ripe berry) & $\begin{array}{l}\text { XM_002264953.2, XM_002278263.2, XM_002269257.2, XM_003634025.1, } \\
\text { XM_003634026.1, XM_003634021.1, XM_003634022.1, XM_003634019.1, } \\
\text { XM_003634028.1, XM_003634023.1, XM_003634024.1, XM_003634027.1 }\end{array}$ \\
\hline \multirow[t]{2}{*}{ Stilbene synthase 5 -like } & 4 (ripe berry) & XM_002268720.2,XM_002278318.2, XM_002263999.2, XM_002269350.2, \\
\hline & ND & XM_002263927.1 \\
\hline Stilbene synthase 6-like & 4 (ripe berry) & XM_002262908.2,XM_002263771.2,XM_003634016.1 \\
\hline \multirow[t]{4}{*}{ Chalcone synthase } & 1 (young berry) & XM_002276885.2, XM_002276910.1 \\
\hline & 2 (low at veraison) & XM_002263983.1 \\
\hline & NC & XM_002276617.1 \\
\hline & ND & XM_002276606.1, XM_002269415.2, XM_003634008.1 \\
\hline
\end{tabular}

Clustering of genes involved in phenylpropanoid metabolism and stilbene biosynthesis. NC, expressed but not clustered; ND, not detected; a transcripts with the highest sequence similarity to the functionally characterised resveratrol synthase, Vst1 [65]; $\mathbf{b}$ transcripts with the highest sequence similarity to the functionally characterised resveratrol synthase, StSy [64].

were unique, even when RPKM counting was performed at $99 \%$ (data not shown), suggesting that the strong coregulation of this gene family was not an artefact of the read mapping process. Eight STSs are represented by probesets on the GeneChip microarray platform, and DeLuc et al. (2011) demonstrated that each of these was up-regulated to some degree late in the growing season, with highest expression from five weeks after veraison until harvest [66]. They also demonstrated that this upregulation was increased in water deficit conditions, so it is possible that the environmental conditions during the season under investigation here could have contributed to, or been the cause of, the highly coordinated upregulation of STSs in ripe berries. A more recent investigation into stilbene synthase expression during grape ripening with the latest and most comprehensive microarray platform showed low expression of STSs in all stages of berry development until post-harvest [67], suggesting that the precise timing of berry harvest could be a vital determinant in stilbene, and thus resveratrol, content in wine. We investigated the expression levels to two STSs across our four developmental stages via quantitative RT-PCR, including the single STS that was located in cluster 8 (XM_003634009.1), and one of the STSs located in cluster 4 due to its specific expression in ripe berries (XM_003634018.1). This PCR-based method validated the result observed from our RNASeq data, and demonstrated that the results were consistent amongst the three biological replicates analysed (Figure 4). 


\section{Differential expression of aroma-related transcripts}

Aroma is an important determinant of wine quality, and the precursors of many aroma compounds found in wine are synthesised during berry development. Compounds from the terpenoid class of biochemicals have been shown to influence the aroma of wine, with several 10-carbon monoterpenes affecting the fruity character of wine [68], and a 15-carbon sesquiterpenoid being responsible for the peppery aroma of Shiraz [69,70]. Monoterpenes are formed through the action of terpene synthase-a (TPS-a; [29]) enzymes that use geranyl pyrophosphate as a substrate, arising from products of the deoxy xylulose-5phosphate (DXP) pathway, isopentenyl pyrophosphate (IPP) and dimethylallyl pyrophosphate (DMAPP). The DXP pathway consists of seven chloroplast-localised enzymes [71], for which six of the encoding transcripts were expressed at all four stages of berry development with little differential regulation. The transcript encoding the final enzyme of the DXP pathway, hydroxymethylbutenyl diphosphate reductase (XM_002284623.2) was in cluster 7 and therefore up-regulated at veraison and in ripe berries (Table 7). Although transcripts encoding components of the DXP pathway were expressed during berry development, we detected almost no expression of putative monoterpene synthases. Similar to the number of putative TPS-a genes identified by Martin et al. (2010) [29], 29 potential monoterpene synthases were found in the RefSeq mRNA collection, all of which were annotated by sequence similarity as myrcene or linalool synthases. Of these 29 transcripts, 26 were not detected in any of the four developing grape samples investigated here. The other three were detected at relatively low transcript abundances $(\mathrm{RPKM}<5)$, with one each in clusters 1 and 10 , and one expressed in the first two stages but not assigned to a cluster (XM_002275786.2, XM_002276009.1 and XM_003634850.1, respectively). The specific expression of XM_002275786.2 in immature green berries when compared with berries at veraison or harvest was confirmed by quantitative RT-PCR (Figure 4). Given the high transcriptome coverage observed in each sample and therefore our ability to detect transcript expression at extremely low levels, this is a strong indication that TPS-a enzymes do not play an important metabolic role for $V$. vinifera (cv. Shiraz) during ripening. In contrast to the

Table 7 Terpenoid pathway transcripts

\begin{tabular}{|c|c|c|}
\hline Encoded protein description & Cluster & RefSeq accession(s) \\
\hline $\begin{array}{l}\text { 4-hydroxy-3-methylbut-2-enyl } \\
\text { diphosphate reductase }\end{array}$ & $\begin{array}{l}7 \text { (veraison } \\
\text { onwards) }\end{array}$ & XM_002284623.2 \\
\hline \multirow[t]{4}{*}{ TPS-a (monoterpene synthases) } & 1 (young berry) & XM_002275786.2 \\
\hline & 2 (decreasing) & XM_002276009.1 \\
\hline & NC & XM_003634850.1 \\
\hline & ND & $\begin{array}{l}\text { XM_003633271.1, XM_003635303.1, XM_002265375.2, XM_003633272.1, XM_003634832.1, } \\
\text { XM_003634833.1, XM_002275070.1, XM_003634834.1, XM_003634838.1, XM_002267425.2, } \\
\text { XM_002267123.1, XM_003634855.1, XM_002274758.2, XM_003635585.1, XM_003634831.1, } \\
\text { XM_002267417.1, XM_003634835.1, XM_003634836.1, XM_003634837.1, XM_003634854.1, } \\
\text { XM_002266772.1, XM_002266983.2, XM_002275237.1, XM_002279833.2, XM_003635411.1, } \\
\text { XM_003635502.1 }\end{array}$ \\
\hline \multirow{2}{*}{$\begin{array}{l}\text { Hydroxymethylglutaryl-coenzyme } \\
\text { A reductase }\end{array}$} & 9 (decreasing) & XM_002275791.2,XM_002265602.1 \\
\hline & NC & XM_002283147.2 \\
\hline Farnesyl pyrophosphate synthase & 9 (decreasing) & XM_002272605.2 \\
\hline \multirow[t]{5}{*}{ TPS-b (sesquiterpene synthase) } & 1 (young berry) & XM_003634648.1,XM_002282960.2,XM_002263544.2 \\
\hline & $\begin{array}{l}2 \text { (early } \\
\text { veraison) }\end{array}$ & XM_002282452.1 \\
\hline & 4 (ripe berry) & XM_002275344.2,XM_002274745.2, XM_002274409.2,XM_002275372.2,XM_002283034.1 \\
\hline & $\begin{array}{l}6 \text { (veraison up- } \\
\text { regulated) }\end{array}$ & XM_002276330.2 \\
\hline & ND & $\begin{array}{l}\text { XM_003634900.1, XM_003634901.1, XM_002275315.1, XM_002277227.2, XM_002273588.2, } \\
\text { XM_002277315.2, XM_002275101.2, XM_002275554.2, XM_002275022.1, XM_002285472.1, } \\
\text { XM_002283040.2, XM_002283308.1, XM_003634597.1 }\end{array}$ \\
\hline \multirow[t]{3}{*}{ Carotenoid cleavage dioxygenase } & 4 (ripe berry) & XM_002268368.2 \\
\hline & $\begin{array}{l}7 \text { (veraison } \\
\text { onwards) }\end{array}$ & XM_002278714.2,XM_002278592.2, XM_002270125.1 \\
\hline & ND & $\begin{array}{l}\text { XM_002281203.1, XM_002274162.1, XM_002269502.2, XM_002281297.2, XM_003631732.1, } \\
\text { XM_003633051.1 }\end{array}$ \\
\hline
\end{tabular}


absence of expression of monoterpene synthases in ripening Shiraz berries, the expression of a linalool/nerolidol synthase was recently found to be highest during veraison in the Gewürztraminer grape variety [72]. Additionally, although significant levels of monoterpenes such as geraniol, linalool and $\alpha$-terpineol are found in Muscat grapes [73] and to a lesser extent in Gewürztraminer and Riesling varieties [72,74], they have not been found at significant levels in red grape varieties. The low level of expression of three putative monoterpene synthases in the earliest Shiraz berry sample (E-L 31) could be a reflection of transcriptional events that were up-regulated during flowering, when monoterpene synthases have been shown to be transcribed [75]. In the absence of monoterpene synthase expression in ripening berries, the presence of transcripts encoding the DXP pathway can be explained by the potential utilisation of IPP and DMAPP for the biosynthesis of other terpene-based metabolites such as carotenoids and phytosterols.

Sesquiterpenes are produced by members of the TPS-b enzyme family from farnesyl pyrophosphate (FPP), which is formed in the cytoplasm from IPP and DMAPP. Cytoplasmic IPP and DMAPP are produced by the mevalonate pathway, consisting of six enzymes for which transcripts were found in each of the four developmental stages. From the mevalonate pathway, two of the three transcripts encoding isoforms of hydroxymethylglutaryl-coenzyme A reductase (HMGR) were in cluster 9 (decreasing expression through development), as was FPP synthase, while all other transcripts were unclustered. HMGR is considered to be the rate limiting enzyme in the mevalonate pathway [76], and thus its up-regulation early in development could indicate a greater requirement for terpene precursors in immature berries. We identified 23 transcripts encoding putative TPS-b enzymes, which are currently annotated by NCBI as valencene synthase-like or germacrene synthase-like genes. The differential regulation of TPSa and TPS-b transcripts in grapes has not previously been reported in detail in microarray experiments due to poor coverage of the TPS gene family by the available probes. For example, on the Affymetrix GeneChip there is only a single probe that interrogates a TPS-a transcript and four probes that interrogate TPS-b transcripts (Additional file 1). In our analysis, however, 10 of the 23 TPS-b transcripts were detected in at least one sample, and all 10 exhibited differential expression during grape development (Table 7). Three transcripts were in cluster 1 , and were therefore specifically expressed in young berries (XM_003634648.1, XM_002282960.2 and XM_002263544.2), two were specifically expressed around veraison and allocated to clusters 2 and 6 (XM_002282452.1 and XM_002276330.2, respectively), and five transcripts were in cluster 4 and up- regulated in ripe berries (XM_002275344.2, XM_002274745.2, XM_002274409.2, XM_002275372.2 and XM_002283034.1).
Remarkably, all of these transcripts except XM_002276330.2 were predominantly expressed in only one of the four samples, demonstrating the existence of tightly controlled differential regulation. Given the importance of some sesquiterpenoids for the aroma of wine (e.g. [69]), members of the TPS-b clade of terpene synthases for which transcripts are up-regulated in ripening berries may be interesting future targets for functional characterisation. We validated the observed differential expression for two transcripts from cluster 1 and two from cluster 4 using qRT-PCR. For three of these transcripts, we confirmed that the extremely specific temporal expression was consistent amongst three biological samples, while in the case of transcript XM_002283034.1, it was relatively highly expressed at the late-veraison stage as well as ripe berries, in one of the three biological replicates (Figure 4).

Another class of potential aroma compounds that stem from terpene metabolic pathways are the $\mathrm{C} 13$ norisoprenoids, such as $\beta$-damascenone and ionone, which are derived as breakdown products of $\mathrm{C} 20$ carotenoids [77]. The breakdown of carotenoids into norisoprenoids is thought to be catalysed by carotenoid cleavage dioxygenase (CCD) enzymes, one of which has been functionally characterised in grapes (VvCCD1) [78]. The transcript encoding VvCCD1, XM_002278714.2, was grouped in cluster 7 , and was highly abundant (RPKM $>200)$ from early-veraison through ripening, while a close homologue XM_002278592.2 was expressed at much lower level but followed a similar expression pattern (Table 7). Transcripts encoding two other putative CCDs were detected in our samples, including XM_002270125.1, which was also grouped in cluster 7, and XM_002268368.2, which was in cluster 4 and highly up-regulated in ripe berries. This last observation is in agreement with a recent microarray study by Guillaumie et al. (2011), who reported that XM_002268368.2 expression increased approximately 2fold in the final week of ripening [13]. Our data therefore provides an indication that the production of $\mathrm{C} 13$ norisoprenoids by the CCD-catalysed enzymatic cleavage of carotenoids is initiated at veraison and continues through until harvest, and could explain the physiological observation that $\beta$-damascenone accumulates after veraison [79].

\section{Conclusions}

RNA-Seq analysis of transcript abundances during berry development has enabled us to carry out a global investigation of gene expression at four time-points in developing grapes and has facilitated a comprehensive description of differential transcriptional events that occurred within a single season for the important wine grape variety $V$. vinifera (cv. Shiraz). We have reported a detailed description of the expression profiles of 23,720 mRNA transcripts contained within the NCBI RefSeq $V$. vinifera collection, and shown that this is an accurate 
reference for transcript abundance measurements. We used gene clustering and the enrichment of Gene Ontology terms to describe the overall biological processes that were regulated during development, and described the transcriptional patterns of genes involved in organic acid, stilbene and terpenoid metabolism as examples of co-regulated and differentially expressed gene families. Quantitative real-time PCR was used to confirm the differential expression patterns observed for 12 of the genes reported, and it was demonstrated that the results obtained with RNA-Seq were consistent with the average expression from three biological replicates. Whether the differential regulation of gene expression described here occurred solely as a consequence of berry development, or in response to specific environmental, biotic or abiotic conditions requires further confirmation during other seasons and in different locations. Also, the extent to which the differential regulation of genes reported here is applicable to other $V$. vinifera varieties is yet to be shown, and the investigation of transcriptional changes at more closely spaced developmental stages will provide further valuable information. Our full transcript abundance analysis, presented in Additional file 1, represents an invaluable resource for hypothesis development and candidate gene selection.

\section{Methods}

\section{Sample collection and berry developmental measurements}

$V$. vinifera (cv. Shiraz) bunches of vines grown at the Nuriootpa Research vineyard, Barossa Valley, South Australia, were tagged at 50\% cap-fall. Three replicates of 20 berries were harvested throughout the 2010-11 season between 9-10 am. Individual replicates consisted of berries from different vines, and each replicate consisted of two berries taken from random positions on each of ten bunches on that vine. Harvesting was carried out by cutting through the pedicel at the junction between stem and berry, frozen immediately in liquid nitrogen, and subsequently stored at $-80^{\circ} \mathrm{C}$ until required. For the purposes of total soluble solids (TSS) estimation, additional fruit (12 berries per sample) was collected and individual berries analysed for ${ }^{\circ} \mathrm{Bx}$ with a digital Pocket Refractometer (Atago, Tokyo). Developmental stages were characterised by changes in berry weight accumulation, TSS and malic and tartaric acid concentration as well as observed changes in berry colour and deformability.

For determination of malic acid and tartaric acid content, each replicate of 20 frozen whole berries was ground to a fine powder in a liquid nitrogen-cooled A11 basic mill (IKA, Germany). Organic acids were extracted from $0.3 \mathrm{~g}$ of powder in $0.5 \mathrm{M}$ ortho-phosphoric acid, $\mathrm{pH} 1.5$, in a final volume of $1.5 \mathrm{ml}$. Samples were mixed for 1 hour at room temperature and centrifuged at
$16,000 \mathrm{~g}$ for $10 \mathrm{mins}$. The supernatant was passed through a $45 \mu \mathrm{m}$ PVDF $30 \mathrm{~mm}$ filter and malic and tartaric acids were quantified using reversed phase HPLC on an Agilent 1100 series HPLC (Agilent Technologies, Santa Clara, USA). The extract $(20 \mu \mathrm{l})$ was injected into a Kinetex $^{\mathrm{TM}} 2.6 \mu \mathrm{m}$ C18 $100 \AA$ column $(150 \mathrm{~mm} \times 4.6$ mm ID) with guard column (Phenomenex, Sydney, Australia), maintained at $30^{\circ} \mathrm{C}$. The mobile phase was 10 $\mathrm{mM} \mathrm{KH_{2 }} \mathrm{PO}_{4}(\mathrm{pH} 2.9)$ with a flow rate of $0.5 \mathrm{ml} / \mathrm{min}$. Detection was carried out at $210 \mathrm{~nm}$ with a photodiode array detector, and concentrations were determined according to calibration curves of appropriate standards using Chemstation for LC 3D systems software (Agilent Technologies, Santa Clara, USA).

\section{RNA extraction and sequencing}

For large scale RNA extraction for next generation sequencing, approximately $2 \mathrm{~g}$ of powder from one of the replicates of harvested berries was ground further with a mortar and pestle and added to $15 \mathrm{ml}$ RNA extraction buffer [80], pre-warmed to $65^{\circ} \mathrm{C}$, consisting of $2 \%$ (w/v) cetyltrimethylammonium (CTAB), 2\% (w/v) polyvinylpyrrolidone (PVP) K-20, $100 \mathrm{mM}$ TRIS-HCL (pH 8.0), $25 \mathrm{mM}$ EDTA, $2.0 \mathrm{M} \mathrm{NaCl}, 0.5 \mathrm{~g} \mathrm{l}^{-1}$ spermidine, with $2 \%(\mathrm{v} / \mathrm{v}) 2$-mercaptoethanol added immediately prior to use. Samples were mixed by vortexing and incubated at $65^{\circ} \mathrm{C}$ for $10 \mathrm{~min}$ with gentle mixing every $3 \mathrm{~min}$, prior to the addition of $10 \mathrm{ml}$ 24:1 chloroform-isoamylalcohol (CIA). Samples were centrifuged at $10000 \mathrm{~g}$ for $10 \mathrm{~min}$ at room temperature, and the top aqueous layer was transferred to fresh tubes. Washes were repeated twice with $10 \mathrm{ml} \mathrm{CIA}$ and $4 \mathrm{ml} 10 \mathrm{M} \mathrm{LiCl}$ was added to the final aqueous layer, which was incubated overnight at $4^{\circ}$ C. Samples were centrifuged at $10000 \mathrm{~g}$ for $30 \mathrm{~min}$ at $4^{\circ}$ $\mathrm{C}$, the supernatant was removed and the pellet was resuspended in $800 \mu \mathrm{l}$ STE buffer containing $1 \mathrm{M} \mathrm{NaCl}$, $10 \mathrm{mM}$ TRIS pH 8.0 and $1 \mathrm{mM}$ EDTA pre-heated to $65^{\circ}$ $\mathrm{C}$. The resuspended RNA was washed once with $800 \mu \mathrm{l}$ $\mathrm{CIA}$ and the aqueous layer transferred to a fresh tube. For a final RNA precipitation, $300 \mu$ l cold isopropanol and $300 \mu \mathrm{l}$ of salt solution containing $1.2 \mathrm{M}$ sodium citrate and $0.8 \mathrm{M} \mathrm{NaCl}$ were added, samples were incubated at $-20^{\circ} \mathrm{C}$ for $10 \mathrm{~min}$, and centrifuged at $10000 \mathrm{~g}$ for $20 \mathrm{~min}$ at $4^{\circ} \mathrm{C}$. The supernatant was discarded, and 1 $\mathrm{ml}$ cold ethanol was added to the pellet. Samples were centrifuged once more at $10000 \mathrm{~g}$ for $10 \mathrm{~min}$, and the supernatant was removed. The pellet was air dried, and resuspended in $100 \mu \mathrm{l}$ DEPC (diethylpyrocarbonate) treated water. RNA integrity and concentration were determined using a Nanodrop 2000 (Thermo Scientific), and samples were diluted to approximately $300{\mathrm{ng} \mathrm{Il}^{-1}}^{-}$ in TE buffer. RNA for qRT-PCR analysis of the other two biological replicates was extracted by the same 
method, except $200 \mathrm{mg}$ of ground grape tissue was used and volumes were adjusted accordingly.

Illumina RNA sequencing was carried out at the Australian Genome Research Facility (AGRF, Melbourne, Australia) on an Illumina HiSeq 2000 instrument (Illumina). RNA quality control was carried out on a 2100 Bioanalyzer (Agilent Technologies) and each sample received an RNA integrity numbers (RIN). Poly(A) mRNA was prepared and sequences from each of the four developmental stages were indexed with unique nucleic acid identifiers. Sequencing on the HiSeq 2000 was carried out according AGRF protocols and following the manufacturer's instructions for the generation of singleend reads, and data was generated with CASAVA 1.8.1 pipeline (Illumina). The sequence reads from all four samples were analysed according to AGRF quality control measures; adaptor or contaminant sequences were removed and reads containing long stretches of ambiguous characters were clipped.

\section{Sequencing data analysis}

For mapping sequence reads against the most recently curated non-redundant mRNA transcriptome, 23720 sequences of $V$. vinifera RefSeq mRNAs [33] were retrieved from the National Centre for Biotechnology Information (http://ncbi.nlm.nih.gov) and CLC Genomic Workbench 4.8 (CLC Bio) was used to assemble the cleaned sequence data against this single reference file in FASTA format. Prior to transcriptome mapping, two nucleotides were trimmed from each end of each sequence read, and reads under 60 nucleotides in length or with greater than two ambiguous nucleotides were not included in the mapping or counting. For inclusion in the calculation of RPKM values, cut-offs were set such that greater than $50 \%$ of a read in contiguous nucleotides must have aligned to a reference transcript with greater than $98 \%$ identity. When reads could be mapped to multiple reference locations, they were assigned to reference transcripts proportionally based on the relative number of unique reads already mapped to each of the reference sequences.

\section{Quantitative real-time polymerase chain reaction}

Quantitative RT-PCR was carried out on cDNA generated from three biological replicates harvested as described above, one of which corresponded to the sample subjected to Illumina sequencing for RNA-Seq analysis. Reactions were set up in KAPA SYBR Fast qPCR Universal ReadyMix (Geneworks, Adelaide, Australia) according to manufacturer's instructions, with genespecific primers $(0.125 \mu \mathrm{M})$ in a final volume of $20 \mu \mathrm{l}$. Details on gene annotations, accessions and primer sets are included in Additional file 3. Thermal cycling conditions involved an initial $95^{\circ} \mathrm{C}$ melt $(3 \mathrm{~min})$, followed by
40 cycles of $95^{\circ} \mathrm{C}(3 \mathrm{~s})$ and $60^{\circ} \mathrm{C}(30 \mathrm{~s})$. Assays were conducted with a C1000 Thermal Cycler fitted with a CFX96 Real-time PCR detection system (BioRad), and analysed using the CFX Manager software (BioRad). Primer pairs were designed to target unique regions of the genes of interest, and PCR and agarose gel analyses were used to verify the absence of non-specific amplification prior to qRT-PCR. Additionally, following reactions DNA melt curves were created for each primer combination to confirm the presence of a single product. The average of two technical repeats was used for each reaction, and the standard error of the mean was calculated for the three biological replicates. Transcripts were normalized to a reference number derived from transcript levels of three reference genes; namely ankyrin-repeat domain protein, elongation initiation factor (eIF-2B) and calcineurin B-like protein.

\section{Global comparison of transcript expression between technical platforms}

Relevant publically available grape Affymetrix probesets were retrieved from http://www.affymetrix.com and microarray raw data were downloaded from PlexDB [81]. Raw CEL files were processed using RMAExpress software (http://rmaexpress.bmbolstad.com) using the background-adjusted and quantile normalised setting, and intensity data was summarised using robust multiarray average (RMA) expression values. Cross-hybridizing probesets (represented by the _s_, _x _ and _ a identifiers) were removed and BLASTn analysis of the express sequence tags (ESTs) on which the remaining probesets were designed was conducted against the current NCBI RefSeq $V$. vinifera mRNA dataset using PERL. Only probesets for which the ESTs matched a RefSeq transcript with an e-value of zero were considered, and where more than one probeset matched to a single RefSeq transcript only the most closely correlating probeset was included. The signal intensities for probesets fulfilling the above requirements were log2 transformed and equivalent expression values from RNA-seq were obtained by calculating $\log 2(\mathrm{RPKM}+1)$ to avoid taking the log of zero. Spearman correlation coefficients between global relative expression and individual transcript expression patterns were calculated using Microsoft Excel for each developmental stage, and an average of the four stages is presented. While Pearson product-moment correlation coefficients yielded similar results, Spearman coefficients were reported due to the non-linear relationship between microarray intensity and absolute expression [82]. When removing transcripts that were not considered expressed from our datasets, probesets with intensities below the $25^{\text {th }}$ percentile (corresponding to a normalised intensity of 4.0) and transcripts with an RPKM $<0.5$ were discarded. 


\section{Cluster analysis and gene ontology assignment}

Clustering of transcript expression patterns based on NCBI RefSeq RPKM levels was carried out with Cluster 3.0 [83]. Prior to cluster formation, transcripts that had an RPKM value below 0.5 in each stage were discarded. RPKM expression values for each transcript were normalised to between -1.0 and 1.0 by multiplying by a scale factor such that the sum of the squares of the four values for each transcript was 1.0. The normalised expression values for each transcript were then centred on zero by subtracting the mean of the four values from each data point so that the mean of each row was zero. Transcripts that displayed a difference of less than 0.5 between the maximum and minimum normalised data points were filtered to select for genes displaying a significant degree of differential regulation. Clustering was carried out using the k-means method for 20 clusters and with the Euclidean similarity metric. After 1000 iterations the reported clustering result was found three times (details can be found in the Cluster 3.0 manual; [83]). RefSeq accessions were compiled from each cluster and their corresponding Genoscope ( $8 \mathrm{x}$ assembly) accession, if available, were input into the AgriGO agricultural gene ontology (GO) analysis tool (http://bioinfo. cau.edu.cn/agriGO/analysis.php) to elucidate enriched GO terms within the cluster when compared with GO terms in the complete $V$. vinifera transcriptome [34]. The REVIGO web server (http://revigo.irb.hr/) was used to summarise the biological processes represented in the lists of significantly enriched GO terms from each cluster [49]. Only Biological Process GO terms with a false discovery rate (FDR; e-value corrected for list size) of $\leq$ 0.05 were submitted to the REVIGO tool, and the "small allowed similarity" setting was selected to obtain a compact output of enriched biological processes. The overall significance of enriched biological processes was expressed as the sum of $100 \mathrm{x}-\log _{10}(\mathrm{FDR})$ for each enriched GO term counted within that process, giving an arbitrary value proportional to the relative statistical significance at which the biological process was enriched. For example, using this technique a biological process including a single enriched term with a FDR of 0.01 would give a value of 200 , while an FDR of $1 \times 10^{-10}$ would give 1000. This technique was adapted from the method used to visualise enriched GO terms as a percentage of the total enriched terms in the TreeMap function of the REVIGO web server [49].

\section{Additional files}

Additional file 1: Absolute expression levels for all NCBI RefSeq transcripts in stages EL 31, 35, 36 and 38 of developing Shiraz grape. Additional file 1 consists of three tables in Microsoft Excel format. Table 1 contains RPKM data for all NCBI RefSeq Vitis vinifera transcripts at developmental stages E-L 31, E-L 35, E-L 36 and E-L 38, and includes their NCBI putative functional annotation, and the closest matching Genoscope accession and Affymetrix Probeset ID for the purpose of cross-referencing to other work. Each Probeset ID is listed once only, and matches to its most similar RefSeq transcript. The cluster to which each gene has been allocated with regards to Figure 3 is also shown. Table 2 contains transcripts expressed with an RPKM of greater than 200 at all four stages of berry development under investigation. Corresponding Genoscope annotations and Affymetrix GeneChip probeset IDs have been assigned through BLASTx and VitisNet Network or Category functional annotations are taken from [32]. Table 3 contains a complete list of all RefSeq transcripts not detected with at least five unique sequencing reads, or with and RPKM $\geq 0.5$ in any of the samples.

Additional file 2: List of all NCBI RefSeq transcripts specifically upregulated 3-fold or greater at a single developmental stage, or during veraison. Additional file 2 consists of a Microsoft Excel File containing five worksheets, containing lists of transcripts specifically upregulated at each developmental stage, or during both veraison stages. RPKM data are also included, as is the NCBI putative functional annotation.

Additional file 3: Primers used for qRT-PCR analysis presented in tabulated format. Microsoft Excel File containing a single worksheet with primer sequences shown for each of 15 genes, including 3 control genes, alongside the gene annotation and accession number.

\section{Competing interests}

The authors declare that they have no competing interests.

\section{Authors' contributions}

CS coordinated sample collection and physiological analysis, participated in transcriptomic data analysis and carried out the GRT-PCR. DW carried out the computational comparison and statistical analysis of RNA-Seq and microarray data. CMF participated in the design and coordination of the study and assisted in drafting the manuscript. DPD conceived the study, isolated berry RNA, compiled and analysed transcriptome sequencing data and drafted the manuscript. All authors read and approved the final manuscript.

\section{Acknowledgements}

CS, DW and CMF were supported by grants from the Grape and Wine Research and Development Corporation, the University of the Adelaide and Wine 2030. DPD received funding from the European Union Seventh Framework Programme (FP7/2007-2013) under grant agreement 275422, which supported a Marie Curie International Outgoing Fellowship. The authors would like to thank Dr Jake Dunlevy for technical assistance with HPLC measurements and David Contreras Pezoa for his help with the comparison of transcript accessions and microarray probeset IDs.

Received: 9 November 2012 Accepted: 4 December 2012

Published: 11 December 2012

\section{References}

1. Coombe BG: Research on development and ripening of the grape berry. Am J Enol Viticult 1992, 43:101-110.

2. Adams DO: Phenolics and ripening in grape berries. Am J Eno/ Viticult 2005, 57(3):249-256

3. Sweetman C, Deluc LG, Cramer GR, Ford CM, Soole KL: Regulation of malate metabolism in grape berry and other developing fruits. Phytochemistry 2009, 70(11-12):1329-1344.

4. Palliotti A, Silvestroni O, Petoumenou D: Seasonal patterns of growth rate and morphophysiological features in green organs of Cabernet Sauvignon grapevines. Am J Enol Viticult 2010, 61(1):74-82.

5. Liu HF, Wu BH, Fan PG, Li SH, Li LS: Sugar and acid concentrations in 98 grape cultivars analyzed by principal component analysis. J Sci Food Agr 2006, 86(10):1526-1536.

6. Lund ST, Bohlmann J: The molecular basis for wine grape quality-a volatile subject. Science 2006, 311(5762):804-805.

7. Conde C, Silva P, Fontes N, Dias ACP, Tavares R, Sousa MJ, Agasse A, Delro $\mathrm{S}$, Geros $\mathrm{H}$ : Biochemical changes throughout grape berry development and fruit and wine quality. Food 2007, 1:1-22. 
8. Deluc LG, Grimplet J, Wheatley MD, Tillett RL, Quilici DR, Osborne C, Schooley DA, Schlauch KA, Cushman JC, Cramer GR: Transcriptomic and metabolite analyses of Cabernet Sauvignon grape berry development. BMC Genomics 2007, 8:429.

9. Terrier N, Glissant D, Grimplet J, Barrieu F, Abbal P, Couture C, Ageorges A, Atanassova R, Leon C, Renaudin JP, et al: Isogene specific oligo arrays reveal multifaceted changes in gene expression during grape berry (Vitis vinifera L.) development. Planta 2005, 222(5):832-847.

10. Waters DL, Holton TA, Ablett EM, Lee LS, Henry RJ: cDNA microarray analysis of developing grape (Vitis vinifera cv. Shiraz) berry skin. Funct Integr Genomics 2005, 5(1):40-58.

11. Pilati S, Perazzolli M, Malossini A, Cestaro A, Dematte L, Fontana P, Dal Ri A, Viola R, Velasco R, Moser C: Genome-wide transcriptional analysis of grapevine berry ripening reveals a set of genes similarly modulated during three seasons and the occurrence of an oxidative burst at veraison. BMC Genomics 2007, 8:428.

12. Ali MB, Howard S, Chen S, Wang Y, Yu O, Kovacs LG, Qiu W: Berry skin development in Norton grape: distinct patterns of transcriptional regulation and flavonoid biosynthesis. BMC Plant Biol 2011, 11:7.

13. Guillaumie S, Fouquet R, Kappel C, Camps C, Terrier N, Moncomble D, Dunlevy JD, Davies C, Boss PK, Delrot S: Transcriptional analysis of late ripening stages of grapevine berry. BMC Plant Biol 2011, 11:165.

14. Fortes AM, Agudelo-Romero P, Silva MS, Ali K, Sousa L, Maltese F, Choi YH, Grimplet J, Martinez-Zapater JM, Verpoorte R, et al: Transcript and metabolite analysis in Trincadeira cultivar reveals novel information regarding the dynamics of grape ripening. BMC Plant Biol 2011, 11:149.

15. Grimplet J, Deluc LG, Tillett RL, Wheatley MD, Schlauch KA, Cramer GR, Cushman JC: Tissue-specific mRNA expression profiling in grape berry tissues. BMC Genomics 2007, 8:187.

16. Rotter A, Camps C, Lohse M, Kappel C, Pilati S, Hren M, Stitt M, CoutosThevenot P, Moser C, Usadel B, et al: Gene expression profiling in susceptible interaction of grapevine with its fungal pathogen Eutypa lata: extending MapMan ontology for grapevine. BMC Plant Biol 2009, 9:104.

17. Hren M, Nikolic P, Rotter A, Blejec A, Terrier N, Ravnikar M, Dermastia M, Gruden K: 'Bois noir' phytoplasma induces significant reprogramming of the leaf transcriptome in the field grown grapevine. BMC Genomics 2009, 10:460.

18. Figueiredo A, Monteiro F, Fortes AM, Bonow-Rex M, Zyprian E, Sousa L, Pais MS: Cultivar-specific kinetics of gene induction during downy mildew early infection in grapevine. Funct Integr Genomics 2012.

19. Pontin MA, Piccoli PN, Francisco R, Bottini R, Martinez-Zapater JM, Lijavetzky D: Transcriptome changes in grapevine (Vitis vinifera L.) cv. Malbec leaves induced by ultraviolet-B radiation. BMC Plant Biol 2010, 10:224.

20. Pastore C, Zenoni S, Tornielli GB, Allegro G, Dal Santo S, Valentini G, Intrieri C, Pezzotti M, Filippetti I: Increasing the source/sink ratio in Vitis vinifera (cv Sangiovese) induces extensive transcriptome reprogramming and modifies berry ripening. BMC Genomics 2011, 12:631

21. Espinoza C, Vega A, Medina C, Schlauch K, Cramer G, Arce-Johnson P: Gene expression associated with compatible viral diseases in grapevine cultivars. Funct Integr Genomics 2007, 7(2):95-110.

22. Tattersall EA, Grimplet J, DeLuc L, Wheatley MD, Vincent D, Osborne C, Ergul A, Lomen E, Blank RR, Schlauch KA, et al: Transcript abundance profiles reveal larger and more complex responses of grapevine to chilling compared to osmotic and salinity stress. Funct Integr Genomics 2007, 7(4):317-333.

23. Bellin D, Ferrarini A, Chimento A, Kaiser O, Levenkova N, Bouffard P, Delledonne $\mathrm{M}$ : Combining next-generation pyrosequencing with microarray for large scale expression analysis in non-model species. BMC Genomics 2009, 10:555.

24. Fasoli M, Dal Santo S, Zenoni S, Tornielli GB, Farina L, Zamboni A, Porceddu $A$, Venturini $L$, Bicego M, Murino V, et al: The grapevine expression atlas reveals a deep transcriptome shift driving the entire plant into a maturation program. Plant Cell 2012, 24(9):3489-3505.

25. Jaillon O, Aury JM, Noel B, Policriti A, Clepet C, Casagrande A, Choisne N, Aubourg S, Vitulo N, Jubin C, et al: The grapevine genome sequence suggests ancestral hexaploidization in major angiosperm phyla. Nature 2007, 449(7161):463-467

26. Velasco R, Zharkikh A, Troggio M, Cartwright DA, Cestaro A, Pruss D, Pindo M, Fitzgerald LM, Vezzulli S, Reid J, et al: A high quality draft consensus sequence of the genome of a heterozygous grapevine variety. PLoS One 2007, 2(12):e1326.
27. Zharkikh A, Troggio M, Pruss D, Cestaro A, Eldrdge G, Pindo M, Mitchell JT, Vezzulli S, Bhatnagar S, Fontana P, et al: Sequencing and assembly of highly heterozygous genome of Vitis vinifera L. cv Pinot Noir: problems and solutions. J Biotechnol 2008, 136(1-2):38-43.

28. Benjak A, Forneck A, Casacuberta JM: Genome-wide analysis of the "cutand-paste" transposons of grapevine. PLoS One 2008, 3(9):e3107.

29. Martin DM, Aubourg S, Schouwey MB, Daviet L, Schalk M, Toub O, Lund ST, Bohlmann J: Functional annotation, genome organization and phylogeny of the grapevine (Vitis vinifera) terpene synthase gene family based on genome assembly, FLcDNA cloning, and enzyme assays. BMC Plant Biol 2010, 10:226.

30. Mortazavi A, Williams BA, McCue K, Schaeffer L, Wold B: Mapping and quantifying mammalian transcriptomes by RNA-Seq. Nat Methods 2008, 5(7):621-628.

31. Zenoni S, Ferrarini A, Giacomelli E, Xumerle L, Fasoli M, Malerba G, Bellin D, Pezzotti M, Delledonne M: Characterization of transcriptional complexity during berry development in Vitis vinifera using RNA-Seq. Plant Physiol 2010, 152(4):1787-1795.

32. Grimplet J, Cramer GR, Dickerson JA, Mathiason K, Van Hemert J, Fennell AY: VitisNet: "Omics" integration through grapevine molecular networks. PLoS One 2009, 4(12):e8365.

33. Pruitt KD, Tatusova T, Brown GR, Maglott DR: NCBI Reference Sequences (RefSeq): current status, new features and genome annotation policy. Nucleic Acids Res 2012, 40(1):D130-D135.

34. Du Z, Zhou X, Ling Y, Zhang Z, Su Z: agriGO: a GO analysis toolkit for the agricultural community. Nucleic Acids Res 2010, 38(Web Server issue):W64W70.

35. Coombe BG: Adoption of a system for identifying grapevine growth stages. Aust J Grape Wine Res 1995, 1:100-110.

36. Feng $C$, Chen $M, X u$ C, Bai L, Yin X, Allan AC, Ferguson IB, Chen K-S: Transcriptomic analysis of Chinese bayberry (Myrica rubra) fruiet development and ripening using RNA-Seq. BMC Genomics 2012, 13:19.

37. Souvorov A, Kapustin Y, Kiryutin B, Chetvernin V, Tatusova T, Lipman D: Gnomon - NCBI Eukaryotic gene prediction tool. Bethesda MD: National Center for Biotechnology Information; 2010.

38. Trapnell C, Pachter L, Salzberg SL: TopHat: discovering splice junctions with RNA-Seq. Bioinformatics 2009, 25(9):1105-1111.

39. Boss PK, Davies C, Robinson SP: Analysis of the Expression of Anthocyanin Pathway Genes in Developing Vitis vinifera L. cv Shiraz Grape Berries and the Implications for Pathway Regulation. Plant Physiol 1996, 111 (4):1059-1066

40. Sabir A, Unver A, Kara Z: The fatty acid and tocopherol constituents of the seed oil extracted from 21 grape varieties (Vitis spp.). J Sci Food Agr 2012, 92(9):1982-1987.

41. McIntyre LM, Lopiano KK, Morse AM, Amin V, Oberg AL, Young LJ, Nuzhdin SV: RNA-seq: technical variability and sampling. BMC Genomics 2011, 12:293.

42. Vogt $T$, Jones P: Glycosyltransferases in plant natural product synthesis: characterization of a supergene family. Trends Plant Sci 2000, 5(9):380-386.

43. Chervin C, El-Kereamy A, Roustan JP, Latche A, Lamon J, Bouzayen M: Ethylene seems required for the berry development and ripening in grape, a non-climacteric fruit. Plant Sci 2004, 167(6):1301-1305.

44. Chervin C, Deluc L: Ethylene signalling receptors and transcription factors over the grape berry development: gene expression profiling. Vitis 2010, 49(3):129-136.

45. Tsukagoshi $H$, Busch W, Benfey PN: Transcriptional regulation of ROS controls transition from proliferation to differentiation in the root. Cell 2010, 143(4):606-616.

46. Li H, Xu Y, Xiao Y, Zhu Z, Xie X, Zhao H, Wang Y: Expression and functional analysis of two genes encoding transcription factors, VpWRKY1 and VpWRKY2, isolated from Chinese wild Vitis pseudoreticulata. Planta 2010, 232(6):1325-1337.

47. Mzid R, Marchive C, Blancard D, Deluc L, Barrieu F, Corio-Costet MF, Drira N, Hamdi S, Lauvergeat V: Overexpression of VvWRKY2 in tobacco enhances broad resistance to necrotrophic fungal pathogens. Physiol Plant 2007 131(3):434-447.

48. Ashburner M, Ball CA, Blake JA, Botstein D, Butler H, Cherry JM, Davis AP, Dolinski K, Dwight SS, Eppig JT, et al: Gene ontology: tool for the unification of biology. The Gene Ontology Consortium. Nat Genet 2000, 25(1):25-29.

49. Supek F, Bosnjak M, Skunca N, Smuc T: REVIGO summarizes and visualizes long lists of gene ontology terms. PLoS One 2011, 6(7):e21800. 
50. Ojeda H, Deloire A, Carbonneau A, Ageorges A, Romieu C: Berry development of grapevines: Relations between the growth of berries and their DNA content indicate cell multiplication and enlargement. Vitis 1999, 38(4):145-150.

51. Wang W, Vinocur B, Shoseyov O, Altman A: Role of plant heat-shock proteins and molecular chaperones in the abiotic stress response. Trends Plant Sci 2004, 9(5):244-252.

52. Kunkee RE: Some roles of malic-acid in the malolactic fermentation in wine-making. FEMS Microbiol Rev 1991, 88(1):55-72.

53. Emmerlich $V$, Linka N, Reinhold T, Hurth MA, Traub M, Martinoia E, Neuhaus HE: The plant homolog to the human sodium/dicarboxylic cotransporter is the vacuolar malate carrier. Proc Natl Acad Sci U S A 2003, 100(19):11122-11126.

54. Hurth MA, Suh SJ, Kretzschmar T, Geis T, Bregante M, Gambale F, Martinoia E, Neuhaus HE: Impaired pH homeostasis in Arabidopsis lacking the vacuolar dicarboxylate transporter and analysis of carboxylic acid transport across the tonoplast. Plant Physio/ 2005, 137(3):901-910.

55. Wheeler GL, Jones MA, Smirnoff N: The biosynthetic pathway of vitamin C in higher plants. Nature 1998, 393(6683):365-369.

56. Melino VJ, Soole KL, Ford CM: Ascorbate metabolism and the developmental demand for tartaric and oxalic acids in ripening grape berries. BMC Plant Biol 2009, 9:145.

57. Agius F, Gonzalez-Lamothe R, Caballero JL, Munoz-Blanco J, Botella MA, Valpuesta V: Engineering increased vitamin $C$ levels in plants by overexpression of a D-galacturonic acid reductase. Nat Biotechnol 2003, 21(2):177-181

58. DeBolt S, Cook DR, Ford CM: L-tartaric acid synthesis from vitamin C in higher plants. Proc Natl Acad Sci U S A 2006, 103(14):5608-5613.

59. Jeandet P, Douillet-Breuil AC, Bessis R, Debord S, Sbaghi M, Adrian M: Phytoalexins from the Vitaceae: biosynthesis, phytoalexin gene expression in transgenic plants, antifungal activity, and metabolism. J Agric Food Chem 2002, 50(10):2731-2741.

60. Gatto P, Vrhovsek U, Muth J, Segala C, Romualdi C, Fontana P, Pruefer D, Stefanini M, Moser C, Mattivi F, et al: Ripening and genotype control stilbene accumulation in healthy grapes. J Agric Food Chem 2008, 56(24):11773-11785.

61. Baur JA, Sinclair DA: Therapeutic potential of resveratrol: the in vivo evidence. Nat Rev Drug Discov 2006, 5(6):493-506.

62. Deluc LG, Quilici DR, Decendit A, Grimplet J, Wheatley MD, Schlauch KA, Merillon JM, Cushman JC, Cramer GR: Water deficit alters differentially metabolic pathways affecting important flavor and quality traits in grape berries of Cabernet Sauvignon and Chardonnay. BMC Genomics 2009, 10:212.

63. Schwekendiek A, Spring O, Heyerick A, Pickel B, Pitsch NT, Peschke F, de Keukeleire D, Weber G: Constitutive expression of a grapevine stilbene synthase gene in transgenic hop (Humulus lupulus L.) yields resveratrol and its derivatives in substantial quantities. J Agric Food Chem 2007, 55(17):7002-7009.

64. Giovinazzo G, D'Amico L, Paradiso A, Bollini R, Sparvoli F, DeGara L: Antioxidant metabolite profiles in tomato fruit constitutively expressing the grapevine stilbene synthase gene. Plant Biotechnol J 2005, 3(1):57-69.

65. Ingrosso I, Bonsegna S, De Domenico S, Laddomada B, Blando F, Santino A, Giovinazzo G: Over-expression of a grape stilbene synthase gene in tomato induces parthenocarpy and causes abnormal pollen development. Plant Physiol Biochem 2011, 49(10):1092-1099.

66. Deluc LG, Decendit A, Papastamoulis Y, Merillon JM, Cushman JC, Cramer GR: Water deficit increases stilbene metabolism in Cabernet Sauvignon berries. J Agric Food Chem 2011, 59(1):289-297.

67. Vannozzi A, Dry IB, Fasoli M, Zenoni S, Lucchin M: Genome-wide analysis of the grapevine stilbene synthase multigenic family: genomic organization and expression profiles upon biotic and abiotic stresses. BMC Plant Biol 2012, 12:130.

68. Battilana J, Emanuelli F, Gambino G, Gribaudo I, Gasperi F, Boss PK, Grando MS: Functional effect of grapevine 1-deoxy-D-xylulose 5-phosphate synthase substitution K284N on Muscat flavour formation. J Exp Bot 2011, 62(15):5497-5508

69. Wood C, Siebert TE, Parker M, Capone DL, Elsey GM, Pollnitz AP, Eggers M, Meier M, Vossing T, Widder S, et al: From wine to pepper: rotundone, an obscure sesquiterpene, is a potent spicy aroma compound. J Agric Food Chem 2008, 56(10):3738-3744.
70. Siebert TE, Wood C, Elsey GM, Pollnitz AP: Determination of rotundone, the pepper aroma impact compound, in grapes and wine. J Agric Food Chem 2008, 56(10):3745-3748.

71. Rohmer M: The discovery of a mevalonate-independent pathway for isoprenoid biosynthesis in bacteria, algae and higher plants. Nat Prod Rep 1999, 16(5):565-574.

72. Martin DM, Chiang A, Lund ST, Bohlmann J: Biosynthesis of wine aroma: transcript profiles of hydroxymethylbutenyl diphosphate reductase, geranyl diphosphate synthase, and linalool/nerolidol synthase parallel monoterpenol glycoside accumulation in Gewurztraminer grapes. Planta 2012, 236(3):919-929.

73. Wilson B, Strauss CR, Williams PJ: Changes in free and glycosidically bound monoterpenes in developing Muscat grapes. J Agric Food Chem 1984, 32(4):919-924.

74. Wilson B, Strauss CR, Williams PJ: The distribution of free and glycosidically-bound Monoterpenes among skin, juice, and pulp fractions of some white grape varieties. Am J Enol Viticult 1986, 37(2):107-111

75. Lucker J, Bowen P, Bohlmann J: Vitis vinifera terpenoid cyclases: functional identification of two sesquiterpene synthase cDNAs encoding (+)-valencene synthase and (-)-germacrene D synthase and expression of mono- and sesquiterpene synthases in grapevine flowers and berries. Phytochemistry 2004, 65(19):2649-2659.

76. Ro DK, Paradise EM, Ouellet M, Fisher KJ, Newman KL, Ndungu JM, Ho KA, Eachus RA, Ham TS, Kirby J, et al: Production of the antimalarial drug precursor artemisinic acid in engineered yeast. Nature 2006, 440(7086):940-943.

77. Mendes-Pinto MM: Carotenoid breakdown products the-norisoprenoidsin wine aroma. Arch Biochem Biophys 2009, 483(2):236-245.

78. Mathieu S, Terrier N, Procureur J, Bigey F, Gunata Z: A carotenoid cleavage dioxygenase from Vitis vinifera L.: functional characterization and expression during grape berry development in relation to C13norisoprenoid accumulation. J Exp Bot 2005, 56(420):2721-2731.

79. Shure KB, Acree TE: Production of beta-damascenone precursors in cellcultures of vitis-Labruscana Cv Concord grapes. Plant Cell Rep 1994, 13(8):477-480.

80. le Provost G, Herrera R, Paiva JA, Chaumeil P, Salin F, Plomion C: A micromethod for high throughput RNA extraction in forest trees. Biol Res 2007, 40(3):291-297.

81. Dash S, Van Hemert J, Hong L, Wise RP, Dickerson JA: PLEXdb: gene expression resources for plants and plant pathogens. Nucleic Acids Res 2012, 40(Database issue):D1194-D1201.

82. Marioni JC, Mason CE, Mane SM, Stephens M, Gilad Y: RNA-seq: an assessment of technical reproducibility and comparison with gene expression arrays. Genome Res 2008, 18(9):1509-1517.

83. de Hoon MJ, Imoto S, Nolan J, Miyano S: Open source clustering software. Bioinformatics 2004, 20(9):1453-1454.

\section{doi:10.1186/1471-2164-13-691}

Cite this article as: Sweetman et al:: Transcriptome analysis at four developmental stages of grape berry (Vitis vinifera cv. Shiraz) provides insights into regulated and coordinated gene expression. BMC Genomics 2012 13:691.

\section{Submit your next manuscript to BioMed Central and take full advantage of:}

- Convenient online submission

- Thorough peer review

- No space constraints or color figure charges

- Immediate publication on acceptance

- Inclusion in PubMed, CAS, Scopus and Google Scholar

- Research which is freely available for redistribution 